\title{
AlleAlle: Bounded Relational Model Finding with Unbounded Data
}

\author{
Jouke Stoel \\ stoel@cwi.nl \\ CWI, Amsterdam \\ The Netherlands
}

\author{
Tijs van der Storm \\ storm@cwi.nl \\ CWI, Amsterdam \\ University of Groningen, Groningen \\ The Netherlands
}

Jurgen J. Vinju
vinju@cwi.nl
CWI, Amsterdam
TU/e, Eindhoven
The Netherlands

\begin{abstract}
Relational model finding is a successful technique which has been used in a wide range of problems during the last decade. This success is partly due to the fact that many problems contain relational structures which can be explored using relational model finders. Although these model finders allow for the exploration of such structures they often struggle with incorporating the non-relational elements.

In this paper we introduce AlleAlle, a method and language that integrates reasoning on both relational structure and non-relational elements - the data- of a problem. By combining first order logic with Codd's relational algebra, transitive closure, and optimization criteria, we obtain a rich input language for expressing constraints on both relational and scalar values.

We present the semantics of AlLEAlLE and the translation of ALLEALle specifications to SMT constraints, and use the off-the-shelf SMT solver Z3 to find solutions. We evaluate AlleAlle by comparing its performance with KoDKOD, a state-of-the-art relational model finder, and by encoding a solution to the optimal package resolution problem. Initial benchmarking show that although the translation times of AlleAlle can be improved, the resulting SMT constraints can efficiently be solved by the underlying solver.
\end{abstract}

CCS Concepts - Theory of computation $\rightarrow$ Constraint and logic programming; • Software and its engineering $\rightarrow$ Specification languages.

Keywords First order logic, relational algebra, SMT solvers, model finding, constraint problems, constraint optimization problems

\footnotetext{
Permission to make digital or hard copies of all or part of this work for personal or classroom use is granted without fee provided that copies are not made or distributed for profit or commercial advantage and that copies bear this notice and the full citation on the first page. Copyrights for components of this work owned by others than ACM must be honored. Abstracting with credit is permitted. To copy otherwise, or republish, to post on servers or to redistribute to lists, requires prior specific permission and/or a fee. Request permissions from permissions@acm.org.

Onward! '19, October 23-24, 2019, Athens, Greece

(c) 2019 Association for Computing Machinery.

ACM ISBN 978-1-4503-6995-4/19/10 . .\$15.00

https://doi.org/10.1145/3359591.3359726
}

ACM Reference Format:

Jouke Stoel, Tijs van der Storm, and Jurgen J. Vinju. 2019. AlleAlle: Bounded Relational Model Finding with Unbounded Data. In Proceedings of the 2019 ACM SIGPLAN International Symposium on New Ideas, New Paradigms, and Reflections on Programming and Software (Onward! '19), October 23-24, 2019, Athens, Greece. ACM, New York, NY, USA, 16 pages. https://doi.org/10.1145/3359591.3359726

\section{Introduction}

In the last decades relational modeling and model finding has been used to solve problems in a wide range of domains, from security [8], program verification and testing [14, 16], to enterprise modeling [3]. ${ }^{1}$ Since many computational problems have relational structures relational model finding has shown to be a powerful and useful method. But there is also a large class of problems that is not purely relational and requires reasoning over other attributes as well.

Consider for instance, a simple file system. This structure can be naturally expressed as a relational problem. However, adding constraints on properties like the depth or the size of file system nodes is not straightforward, or cannot be solved efficiently. In this paper we propose AlleAlle, a language that allows users to model both the relational and the nonrelational elements -the data- of their problem.

AlleAlle combines first order logic, Codd's relational algebra (projection, restriction, renaming and natural join) [10], and (reflexive) transitive closure in a single formalism. ALLEALLE specifications can be translated to SMT formulas which in turn can be solved by an off-the-shelf SMT solver, such as Z3 [22]. We implemented these ideas in a prototype tool. ${ }^{2}$

Next to solving Constraint Satisfaction Problems (CSP), AlleAlle can be used to solve Constraint Optimization Problems (COP) (cf. traveling salesman). This is achieved by extending the syntax of AlLEAlle with the ability to express optimization objectives on relations. These optimization criteria are added to the translated SMT formulas and can be solved using Z3's built-in optimization solver $v Z$ [7].

We perform an initial performance benchmark and evaluate AlleAlle's expressiveness on a well-known problem in software engineering: optimal package resolution [1]. This

\footnotetext{
${ }^{1}$ For a overview of the different areas where relational model finding has been applied visit http://alloytools.org/citations/case-studies.html. ${ }^{2}$ https://github.com/cwi-swat/allealle
} 
problem, faced by software package managers, can be compactly expressed as a relational problem in ALLEALLE and we show that the resulting SMT formula can be efficiently solved by the underlying SMT solver.

The contributions of this paper can be summarized as follows:

- AlleAlle, a language combining Codd's relational algebra with first order logic, transitive closure, and optimization objectives (Section 3).

- A translation semantics expressed by compiling AlleAlle specifications to SMT constraints (Section 4).

- Initial performance benchmarking of AlleAlle, including a realistic benchmark based on the optimal dependency resolution problem (Section 5).

We conclude the paper with a discussion of related work (Section 6), and an outlook towards future work (Section 7).

\section{Alle Alle}

AlleAlle is an intermediate language similar to KoDKoD's [31] internal model. As such it is aimed at being a target language for high-level relational modeling languages such as Alloy [15, 30]. Instead of using SAT solvers to solve relational constraints, however, AllEAlle leverages native data theories built into SMT solvers, such as Z3. Because of this, AlleAlle can support constraints over unbounded data such as integers, reals, and strings, without having to encode such data values into boolean propositions. As a result, relational specifications employing constraints over data do not suffer from exponential blow-up problems that may occur, for instance, when using fixed bit-width integers in Alloy or KoDKOD. In other words, the solving power of ALLEALle is a super-set of that of KoDKOD.

AlleAlle is designed to be extensible. Our current implementation supports native integer constraints, but the design of the language and the translation to SMT constraints allows support for other theories (e.g., reals, strings, etc.) in the same way as the current prototype supports integer constraints. Below we illustrate how AlleAlle combines Codd's relational algebra and unbounded data constraints using the example of a file system specification.

\subsection{Modeling a File System in Alue Alle}

Imagine that we would like to model a new kind of file system and we want to test our design before building the new system. Our new simple file system would have the following structural constraints: it may contain both directories and files, it only has one root, there can be no cyclic dependencies and everything must be reachable from the root. The file system does not allow symbolic links (preventing cyclic references).

Next to these structural constraints we also have some non structural constraints namely, every file must have a positive size; the size of a directory derives from the size of its contents. Finally, every file and directory has a depth which encodes the distance from the root in the hierarchy.

To check these constraints we create an AlleAlle specification that encodes the above constraints, as shown in Listing 1 . In the next paragraphs we will explain the different parts of this specification.

Declaring relations The first part, lines 1 to 4 , contains the declarations of the relations. Every relation declaration has three parts: the name of the relation, its header, and its tuple bounds. In ALLEALlE all relations are bounded meaning that all the tuples that are potentially part of the relation are defined in its upper bound.

For instance, the File relation on line 1 has three attributes which are defined in its header: oid, depth and size. The attribute oid is of the id domain while depth and size are of the int domain. The id domain is a bounded domain of arbitrary chosen labels, or atoms. The domain contains exactly those values as specified in the relation declarations of the specification. For instance, for this specification the id domain consists of $f \odot, f 1, f 2, d \theta, d 1$ and $d 2$.

The right hand side of the relation declaration lists the tuple bounds. These encode the tuples that can be part of a relation. The file relation contains both a lower bound (the tuple set after the >= sign), and an upper bound (the tuple set after the <= sign). Every relation must have an upper bound. Lower bounds are optional.

Lower bounds can be used to encode partial solutions. They encode the tuples that must be part of every satisfying instance. In our example we see that the lower bound of the File relation has one tuple, 〈oid: f0, depth: 2, size: 100〉. This means that in every satisfying instance found by the solver the relation File must at least contain this tuple. In other words, we specify that our file system always must have the file (identified by) fo with a size of 100 and two steps removed from the root (depth: 2).

The upper bound, on the other hand, contains the tuples that may be part of a satisfying instance. For the File relation this means that two more tuples may be part of any satisfying instance. Both of these tuples contain question marks for the depth and size attributes. These question marks -or holes - in the tuple definition indicate that the value can be freely assigned by the solver as long as the values satisfy the specification. Holes can only be introduced for non-id attributes. Attributes of the id domain always need a value assigned.

The lower and upper bounds of the Root relation (line 3) are equal to each other and contain a tuple set with only one tuple, $\{<d \theta>\}$. When the lower and upper bounds of a relation are equal, the = sign is used to define the exact bound. As a result, in every possible satisfying instance this relation must contain exactly these tuples and not more. The Dir relation only has an upper bound (line 2). This indicates that, 


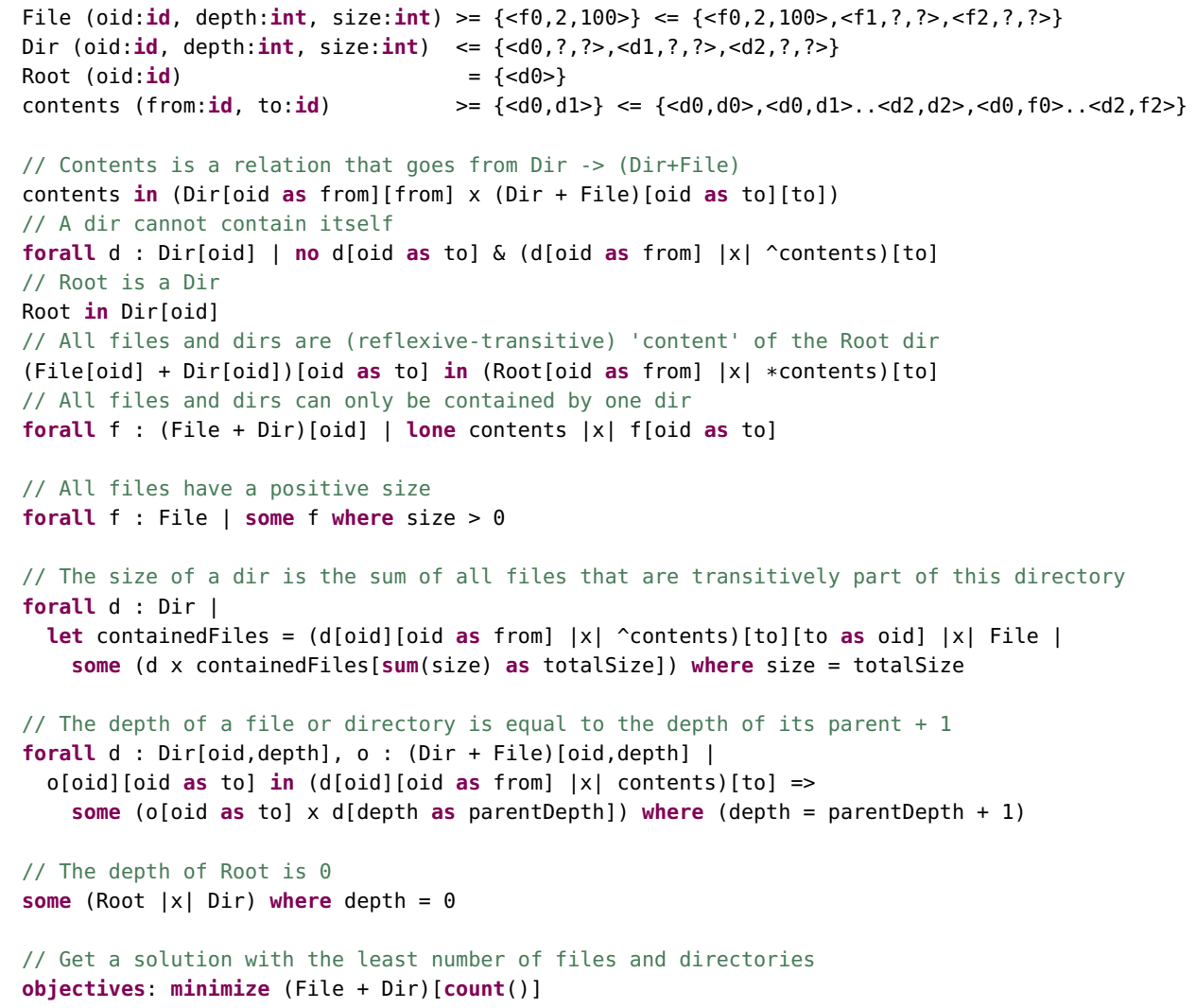

Listing 1. AlleAlle specification of a small file system, original example comes from [28]. [ . . ] is projection, [ . . as ...] is renaming, $\mathrm{x}$ is cartesian product, $\&$ is intersection, + is union, $*$ and $`$ are (reflexive) transitive closure, $|x|$ is natural join.

according to the relation definition, the empty relation is an accepted instance.

The contents relation (line 4) is a binary relation encoding which directories and files are contained by some directory. The .. notation is a short hand notation to define a range of tuples. $^{3}$

Declaring constraints The next part of the specification, lines 6 to 31, describes constraints on the relations. Specifications have no directionality but for clarity of the example we artificially split the constraints in two parts. The first part, lines 6 to 15 , defines constraints on the relational shape of the solution. The second part, lines 17 to 31 , defines constraints on the data.

Line 7 constrains the contents relation to be a subset of the Dir x (Dir + File) relation. This enforces the contents relation to only contain tuples of which the id of the from attribute exists in the Dir relation and the id of the to attribute exists in either the Dir or File relation. Without this constraint the content relation might contain junk. In other words, it might contain tuples tying non-existing directories to other non-existing directories or files. Since the relation

\footnotetext{
${ }^{3}$ The range $\left.\langle\mathrm{d} 0\rangle, .<\mathrm{d} 2\right\rangle$ denotes the tuples $\langle\mathrm{d} \theta\rangle,\langle\mathrm{d} 1\rangle,\langle\mathrm{d} 2\rangle$. Likewise, the range $<\mathrm{d} \theta, \mathrm{f} \theta>\ldots<\mathrm{d} 1, \mathrm{f} 1>$ denotes the tuples $<\mathrm{d} \theta, \mathrm{f} \theta>,<\mathrm{d} \theta, \mathrm{fl}>,<$ $\mathrm{d} 1, \mathrm{f} \Theta>,<\mathrm{d} 1, \mathrm{fl}>$.
}

definition does not state anything on how different relations relate to each other these associations must be supplied as extra constraints.

Union compatibility In Codd's relational algebra the union $(+)$, intersection (\&), difference (-), subset (in), and equality (=) operators require relations to be union compatible with each other. This means that both relations must have the exact same header (both attribute names and associated domains). For instance, on line 11 the constraint that enforces that Root 'is a' Dir is expressed using the subset (in) operator. The header of the root operator on the left hand side only contains the single attribute oid of the id domain. The header of the Dir relation on the other hand has three attributes (oid, depth and size). Since the subset operator needs the relations to be union compatible we use the projection ([ ]) operator on the Dir relation to project the oid attribute out of the Dir relation resulting in a new relation with only one attribute of the same domain.

Transitive closure Line 9 states that no directory can contain itself expressed with the use of the transitive closure operator. Both the transitive closure $(\wedge)$ and reflexive transitive closure $(*)$ are special operators in AlleAlle. They are not part of the traditional relational algebra since it is not 
possible to calculate such a transitive closure on relations in general [2].

Since AlleAlle relations are bounded it is possible to implement both operators, albeit with restriction: both operators only operate on binary relations with two attributes of the id domain. Line 9 applies the transitive closure over the contents relation.

The other constraints in the first part of the specification ensure that all directories and files are reachable from the Root directory (line 13), and that files and directories can only be contained by one directory or none, as is the case for the Root directory (line 15).

The used multiplicity constraints lone and some have their standard semantics: lone means zero or one tuple in the relation is required, some means at least one tuple is required.

Restriction Lines 17 to 31 define data constraints. Line 18 states that all files must have a positive size. To express this constraint the restrict operator (where) is used. Using the restrict operator we can formulate constraints on the attributes of a relation. Applying the restrict operator on a relation results in another relation. To enforce that this restriction holds for all files, the multiplicity constraint some is used. This forces the restricted relation to contain at least one tuple.

Aggregate functions Lines 21-23 define the value of the size attribute of directories. The size of a directory in the file system is the summation of the sizes of the files which are (transitively) contained by the directory. On line 22 the containedFiles relation of the current directory $d$ is defined using the transitive closure of the contents relation which is (naturally) joined $(|x|)$ with the current directory $d$. This relation is then used to calculate the size of the directory by using the aggregation function sum.

The sum function sums up all the values of the size attributes in the containedFiles relation. The result of applying an aggregation function is yet another relation containing zero or one tuples with one attribute (in this case totalsize). Other available aggregation functions include min, max, avg and count. Count is the only aggregation function that does not need an attribute to perform the aggregation on since it counts the number of tuples in the relation. Note that with the use of the count aggregation function all other multiplicity constraints (some, one, lone, no) can be expressed.

The remaining constraints describe the value of the depth attribute (line 26-28) and enforce the depth of the Root directory to be zero (line 31 ).

Optimization objectives The last line, line 34, defines a single optimization objective. This objective states that we

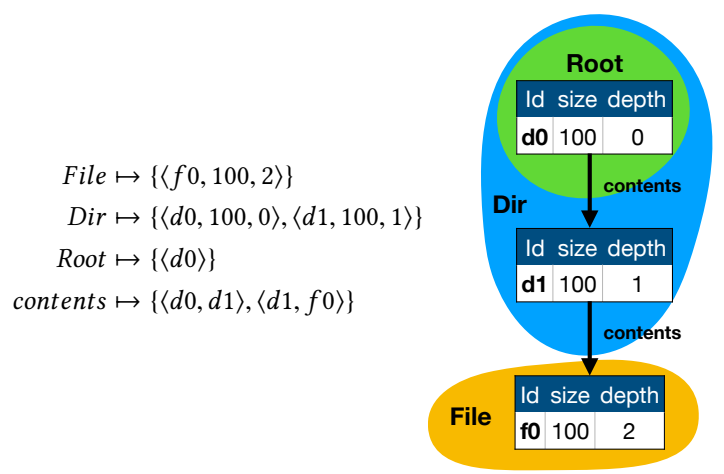

Figure 1. The minimal instance of the small file system specification

want to optimize on the cardinality of the File and Dir relations. Only a relation with a single integer attribute can be used as an optimization criterion.

The optimization criteria are so called "soft constraints". This means that, other than the previously described "hard constraints" (lines 6-31), they do not influence the total number of satisfying instances of a problem. They do influence the order in which the model finder returns solutions. In other words, the instance that is returned first will be the instance that is optimal considering the optimization objectives.

The found solution Figure 1 shows the minimal solution of the file system specification. The found solution contains a binding for all the declared relations. Since the optimization objective stated that we wanted a minimal number of files and directories, it returned a solution that contains only those tuples that were part of our lower bound definition (in the case of File and Root) and those tuples that were needed to get a consistent model according to the described constraints (in case of the Dir and contents relations).

Next to that, the model finder returned a binding for all the introduced holes. The definition of the File tuple <f०, 2, 100> and the constraints on the data determined the values of the other depth and size attributes.

\section{Formal Definition of Alle Alle}

Figure 2 shows the abstract syntax of AlleAlle. We define an AlleAlle problem as follows. A problem $P$ consists of relation definitions $R_{1} \ldots R_{n}$ which are bound to a relational variables $r_{1} \ldots r_{n}$, formulas $F_{1} \ldots F_{n}$ and possibly optimization criteria $O_{1} \ldots O_{n}$. A formula $F$ is a sentence over an alphabet of the relational variables $r_{1} \ldots r_{n}$. A binding $b$ is an instance of all the problem's free relational variables to relational constants. A relational constant of $R$ is a set of tuples. The binding $b$ is said to be a satisfying instance of $P$ if it conforms to the relation definitions of $P$ and makes all the formulas of $P$ true. 


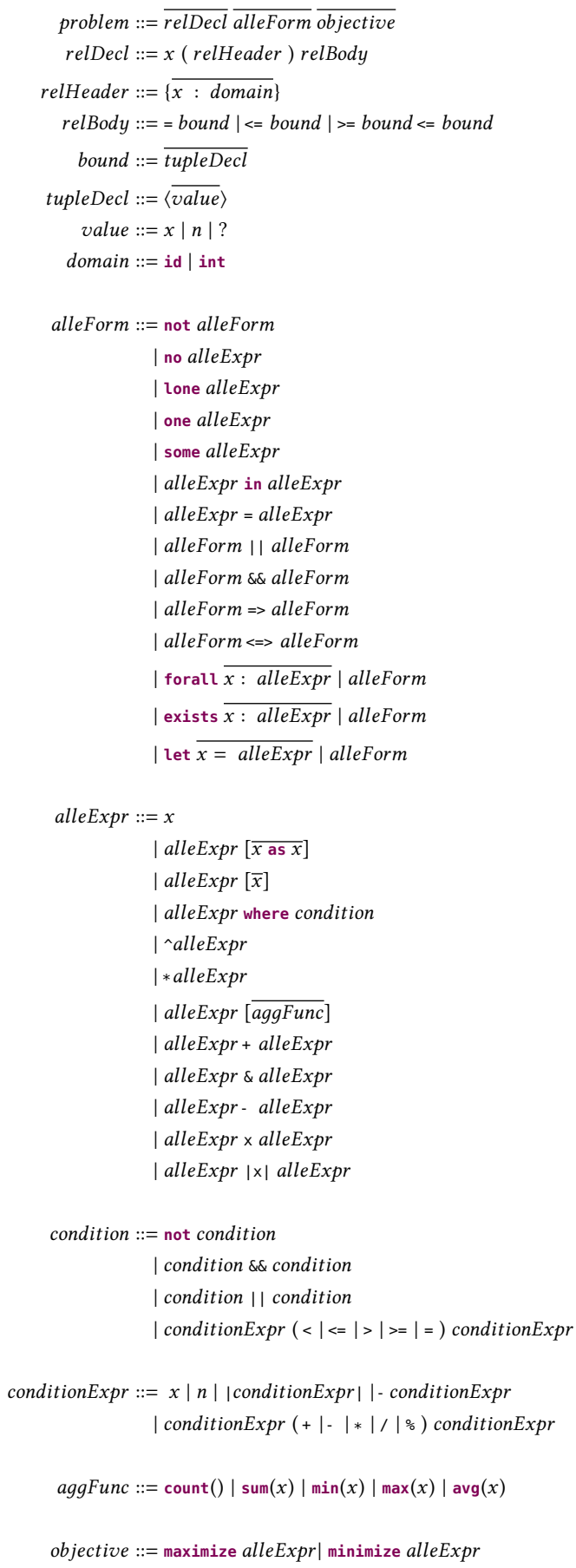

Figure 2. Abstract Syntax of AlleAlle

Relations are defined as in the relational model $[10,12]$. A relation $R$ over multiple domains $D_{1} \ldots D_{n}$, not necessary distinct, consists of a header $H$ and a body $B$. The header $H$ consists of a fixed set of attribute names, domain pairs $\left\{\left\langle a_{1}: D_{1}\right\rangle \ldots\left\langle a_{n}: D_{n}\right\rangle\right\}$. An attribute name is an arbitrary label. A domain is a named set of scalar values, all of the same type. Attribute names in a relation are distinct.
A body $B$ consists of a set of tuples $\left\{T_{1} \ldots T_{n}\right\}$. Since the body is a true set it means that per definition the tuples in the body must be unique. A tuple $T$ consists of a set of attribute name, value pairs $\left\{\left\langle a_{1}: v_{1}\right\rangle \ldots\left\langle a_{n}: v_{n}\right\rangle\right\}$. For each attribute name and domain pair $\left\langle a_{n}: D_{n}\right\rangle$ in $H$ there exists an attribute name and value pair $\left\langle a_{n}: v_{n}\right\rangle$ in $T$ where $v_{n}$ is drawn from $D_{n}$. Relations can be bound to relational variables which are arbitrary labels.

\subsection{Attribute Domains in Alle Alle}

As stated in the definition above, the attribute domains are named sets of scalar values. Currently AlLEAlle supports the int and id domains. The scalars of the int domain are defined by the underlying SMT solver which is the unbounded set of all integer numbers.

The id domain is a bounded domain consisting of arbitrary chosen labels (atoms). Like mentioned earlier, it contains exactly those atoms that are defined in a specification. Please note that the existence of this domain is not strictly essential (since it could be modeled using int values) but it allows for a convenient way to model different dependencies between relations (associations, containment, specialization, etc.).

\subsection{Semantics}

Figure 3 shows the semantics of AlleAlle. We do not include the semantics of the optimization objectives since they are orthogonal to the semantics of formulas and expressions, and defined in terms of the underlying SMT solver, $v Z$ [7]. The meaning of an AlLEAlle problem is defined by four functions, $P, R, F$ and $E$, which can be recursively applied. The function $R$ accepts a relation declaration and binding and returns whether the header of the binding is equal to the header of the declaration and whether the lower bound of the declaration is a subset of the body of the binding which in turn must be a subset of the upper bounds of the declaration. The function $F$ accepts an AlleAlle formula and a binding and returns whether the binding satisfies the formula. The function $E$ accepts an AlleAlle expression and a binding and returns a relational constant. The function $P$ acts as the starting point and accepts a Problem and a binding, and calls the $R$ and $F$ functions.

The semantics of the rename, project, restrict, and aggregate operators on relations are defined in the standard way [10]. The same goes for union $(\cup)$, intersection $(\cap)$, difference $(\backslash)$ and cartesian product $(\times)$. Union, intersection and difference can only be applies on union compatible relations (see 2.1). Cartesian product requires two relations with disjoint headers. Applying the cartesian product on two relations with respectively $n$ and $m$ sized tuples flattens both relations into a new relation of $n+m$ sized tuples.

The semantics of AlleAlle shown in Figure 3 defines the meaning of an ALLEALle problem given an assignment of relation constants to all relational variables in the binding $b$. In order to find solutions rather than check their truth 


\begin{tabular}{|c|c|}
\hline$P$ & $:$ problem $\rightarrow$ binding $\rightarrow$ boolean \\
\hline$R$ & $:$ relDecl $\rightarrow$ binding $\rightarrow$ boolean \\
\hline$F$ & $:$ alleForm $\rightarrow$ binding $\rightarrow$ boolean \\
\hline E & $:$ expr $\rightarrow$ binding $\rightarrow$ constant \\
\hline binding & $:$ var $\rightarrow$ constant \\
\hline$P \llbracket r_{1} \ldots r_{n} f_{1} \ldots f_{m} \rrbracket b$ & $=R \llbracket r 1 \rrbracket b \wedge \ldots \wedge R \llbracket r_{n} \rrbracket b \wedge F \llbracket f_{1} \rrbracket b \wedge \ldots \wedge F \llbracket f_{m} \rrbracket b$ \\
\hline$R \llbracket x(h)[l, u] \rrbracket b$ & $=h=b[x]_{\text {header }} \wedge l \subseteq b[x]_{\text {body }} \subseteq u$ \\
\hline$F \llbracket \operatorname{not} f \rrbracket b$ & $=\neg F \llbracket f \rrbracket b$ \\
\hline$F \llbracket$ no $r \rrbracket b$ & $=|E \llbracket r \rrbracket b|=0$ \\
\hline$F \llbracket$ lone $r \rrbracket b$ & $=|E \llbracket r \rrbracket b| \leq 1$ \\
\hline$F \llbracket$ one $r \rrbracket b$ & $=|E \llbracket r \rrbracket b|=1$ \\
\hline$F \llbracket$ some $r \rrbracket b$ & $=|E \llbracket r \rrbracket b|>0$ \\
\hline$F \llbracket r$ in $s \rrbracket b$ & $=E \llbracket r \rrbracket b \subseteq E \llbracket s \rrbracket b$ \\
\hline$F \llbracket r=s \rrbracket b$ & $=E \llbracket r \rrbracket b \subseteq E \llbracket s \rrbracket b \wedge E \llbracket s \rrbracket b \subseteq E \llbracket r \rrbracket b$ \\
\hline$F \llbracket f \| g \rrbracket b$ & $=F \llbracket f \rrbracket b \vee F \llbracket g \rrbracket b$ \\
\hline$F \llbracket f \& \& g \rrbracket b$ & $=F \llbracket f \rrbracket b \wedge F \llbracket g \rrbracket b$ \\
\hline$F \llbracket f=>g \rrbracket b$ & $=\neg F \llbracket f \rrbracket b \vee F \llbracket g \rrbracket b$ \\
\hline$F \llbracket f \Leftrightarrow \Rightarrow g \rrbracket b$ & $=F \llbracket f \rrbracket \Longleftrightarrow F \llbracket g \rrbracket b$ \\
\hline$F \llbracket$ forall $v_{1}: r_{1} \ldots v_{n}: r_{n} \mid f \rrbracket b$ & $=\wedge_{t \in E \llbracket e_{1} \rrbracket b}\left(F \llbracket\right.$ forall $\left.v_{2}: e_{2} \ldots v_{n}: r_{n} \mid f \rrbracket\left(b \oplus v_{1} \mapsto\{t\}\right)\right)$ \\
\hline$F \llbracket$ exists $v_{1}: r_{1} \ldots v_{n}: r_{n} \mid f \rrbracket b$ & $=\bigvee_{t \in E \llbracket r_{1} \rrbracket b}\left(F \llbracket\right.$ exists $\left.v_{2}: r_{2} \ldots v_{n}: r_{n} \mid f \rrbracket\left(b \oplus v_{1} \mapsto\{t\}\right)\right)$ \\
\hline$F \llbracket$ let $v_{1}: r_{1} \ldots v_{n}: r_{n} \mid f \rrbracket b$ & $=F \llbracket$ let $v_{2}: r_{2} \ldots v_{n}: r_{n} \mid f \rrbracket\left(b \oplus v_{1} \mapsto E \llbracket r_{1} \rrbracket b\right)$ \\
\hline$E \llbracket x \rrbracket b$ & $=b[x]$ \\
\hline$E \llbracket r\left[a_{1}\right.$ as $a a_{1}, \ldots a_{n}$ as $\left.a a_{n}\right] \rrbracket b$ & $=\rho_{\left(a a_{1} / a_{1} \ldots a a_{n} / a_{n}\right)} E \llbracket r \rrbracket b$ \\
\hline$E \llbracket r\left[a_{1} \ldots a_{n}\right] \rrbracket b$ & $=\Pi_{\left(a_{1} \ldots a_{n}\right)} E \llbracket r \rrbracket b$ \\
\hline$E \llbracket r$ where $c \rrbracket b$ & $=\sigma_{c} E \llbracket r \rrbracket b$ \\
\hline$E \llbracket \wedge r \rrbracket b$ & $\begin{aligned}= & \text { let } m \leftarrow E \llbracket r \rrbracket b \text { in }\left\langle m_{\text {header }},\left\{\left(x: i d_{x}, y: i d_{y}\right)\left|\exists i d_{1} \ldots i d_{n}\right|\right.\right. \\
& \left.\left.\left(x: i d_{x}, y: i d_{1}\right),\left(x: i d_{1}, y: i d_{2}\right) \ldots\left(x: i d_{n}, y: i d_{y}\right) \in m_{\text {body }}\right\}\right\rangle\end{aligned}$ \\
\hline$E \llbracket * r \rrbracket b$ & $=E \llbracket \wedge r \rrbracket b \cup \mathbb{I}$ \\
\hline$E \llbracket r[f()] \rrbracket b$ & $=f() E \llbracket r \rrbracket b$ (where $f$ is count) \\
\hline$E \llbracket r[f(a)] \rrbracket b$ & $=f(a) E \llbracket r \rrbracket b($ where $f$ is sum, avg, $\min$ or $\max ))$ \\
\hline$E \llbracket r+s \rrbracket b$ & $=E \llbracket r \rrbracket b \cup E \llbracket s \rrbracket b$ \\
\hline$E \llbracket r \& s \rrbracket b$ & $=E \llbracket r \rrbracket b \cap E \llbracket s \rrbracket b$ \\
\hline$E \llbracket r-s \rrbracket b$ & $=E \llbracket r \rrbracket b \backslash E \llbracket s \rrbracket b$ \\
\hline$E \llbracket r \times s \rrbracket b$ & $=E \llbracket r \rrbracket b \times E \llbracket s \rrbracket b$ \\
\hline$E \llbracket r|\mathrm{x}| s \rrbracket b$ & $=E \llbracket r \rrbracket b \bowtie E \llbracket s \rrbracket b$ \\
\hline
\end{tabular}

Figure 3. Semantics of AlleAlle. Variables $f$ and $g$ range over formulas, $r_{n}$ and $s$ over expressions. The $\oplus$ operator updates bindings. I represents the binary identity relation on all values in the id domain. $\cup, \cap, \backslash$ and $\times$ have their standard relational algebra semantics.

$$
\begin{array}{cc}
\text { form }::=\top|\perp| x \mid \neg \text { form } \mid \text { form } \wedge \text { form } \mid \text { form } \vee \text { form } \mid \\
\quad \operatorname{expr}(<|\leq|=|\geq|>) \text { expr } \\
\text { expr } \quad:=\text { literal }|x| \text { expr }+ \text { expr } \mid \text { expr }- \text { expr } \mid \text { expr } * \text { expr } \mid \\
\quad \text { expr } / \text { expr } \mid \text { expr \% expr } \mid \text { form } \text { expr : expr }
\end{array}
$$

Figure 4. Definition of form and expr.

value, however, AlLEALlE problems are translated to SMT formulas.

\section{Translating AlleAlle to SMT}

Specifications are translated to SMT constraints. Figure 4 describes the definition of the resulting formula (form) that the translation algorithm produces. Our prototype of AlleAlle translates AlleAlle problems to the standard SMT-LIB format, which is supported by multiple SMT solvers [6]. As a result, AlleAlle can potentially be used in combination with different SMT solvers as backends. ${ }^{4}$

Apart from the optimization criteria, the translation consists of flattening AlLEAlle problems to a single SMT formula within the logic fragment of quantifier-free non-linear integer arithmetic (QF-NIA). This means that the final SMT formula is a large, but flat formula made up of negation, conjunction, disjunction, integer arithmetic, (in)equalities and if-then-else constructs, as shown in Figure 4.

Before we go into the details of the translation rules we will give an example of how translation unfolds for a small problem.

\subsection{Translation Example}

Assume we have a relation Person with two attributes pId and age which is defined as follows:

$$
\text { Person ( } p I d: \text { id, age: int) }<=\{<p 1,17>,<p 2, ?>\}
$$

This relation has an upper bound containing two tuples. The lower bound is omitted and thus empty (i.e. the empty set). Consequently, a satisfying instance may hold zero, one or two tuples in the Person relation. The first tuple, $\langle p 1,17>$, assigns the value 17 to the age attribute. This means that if this tuple is present the value of age must be 17 . In the second tuple, $<p 2$, ? $>$, the value of the age is left open meaning that the value is left to the underlying solver.

Next we define the following constraint:

some Person where age $>=18$

This constraint states that there must be at least one person who is an adult. Or more precisely, there needs to be at least one tuple in the Person relation where the value of the age attribute is equal to or greater than 18. Please note that the first tuple in the relation, $\langle p 1,17>$, can never satisfy this constraint since the value of its age attribute will always be 17.

As a first step in the translation an environment $\rho$ is constructed, mapping relation names (e.g., Person) to an internal relation representation. In the example the created environment $\rho$ is as follows:

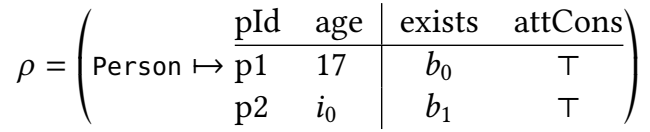

The internal representation of the Person relation consists of a table, with columns for the declared attributes pId and age, and two additional columns, exists and attCons. The pId attribute contains the values p1 and p2 both drawn from the id domain. For the first tuple the age attribute contains the constant 17. This is a consequence of the given relation

\footnotetext{
${ }^{4}$ Currently optimization criteria are not supported by all SMT solvers. At
} least Z3 [7] and MathSAT5 [26] have built-in support. 
definition where the age attribute for this tuple was assigned 17. For the second tuple the age attribute contains an integer variable $i_{0}$. Since in the relation definition this value was left open it is converted to a fresh integer variable.

The exists column encodes whether the tuple should be present in a satisfying instance or not. In this case the value of the exists column for both tuples contains a fresh boolean variable, $b_{0}$ and $b_{1}$ respectively. This is due to the fact that both tuples are part of the upper bound of the relation but not of the lower bound (since the lower bound of this relation is the empty set). For each satisfying instance the solver will assign truth values to these variables. For instance, if $b_{0}=\top$ and $b_{1}=\perp$ it means that the tuple $\langle\mathrm{p} 1,17\rangle$ is in the Person relation but $<\mathrm{p} 2$, ? $>$ is not. The attCons column encodes constraints formulated on the attribute values. Initially the attCons attributes have $T$ assigned.

The next step in the translation is the translation of the constraints. The translation of constraints consists of the recursive application of two translation functions $T_{F}$ for the translation of AlleAlle formulas and $T_{E}$ for the translation of AlleAlle expressions. Their full definitions are shown in Figures 8 and 9. The full translation tree for this example would look like:

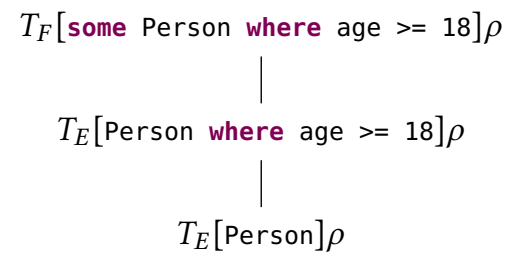

We describe the translation of the example in a bottom-up fashion. The first expression that is translated is the lookup of the Person relation from the environment $\rho$ :

$$
\left.T_{E}[\text { Person }] \rho=\rho \text { (Person }\right)=\begin{array}{ll|cc}
\text { pId } & \text { age } & \text { exists } & \text { attCons } \\
\hline \mathrm{p} 1 & 17 & b_{0} & \mathrm{~T} \\
\mathrm{p} 2 & i_{0} & b_{1} & \mathrm{~T}
\end{array}
$$

As shown above, the result of the translation function $T_{E}$ is another relation. Now the outer where expression is translated as follows:

$T_{E}[$ Person where age $>=18] \rho=$\begin{tabular}{ll|cc} 
pId & age & exists & attCons \\
\hline $\mathrm{p} 1$ & 17 & $b_{0}$ & $\perp$ \\
$\mathrm{p} 2$ & $i_{0}$ & $b_{1}$ & $i_{0} \geq 18$
\end{tabular}

The restriction expression (age $>=18$ ) forces additional constraints on the age attribute. In case of the first tuple the age attribute contains the constant 17 . Since 17 is less than 18 , the value $\perp$ is assigned to the to the attcons attribute. For the second tuple the age attribute was left open which resulted in the introduction of the $i_{0}$ variable. For this tuple the constraint $i_{0} \geq 18$ is added to the attCons column.

The last step is the translation of the outer formula: $T_{F}$ [some Person where age >= 18] $\rho$. The $T_{F}$ function flattens the relation into a flat SMT formula. The translation of the

$$
\begin{array}{ll}
\text { rel } & ::=\left\langle{\overline{x: \text { domain }_{\text {header }},}}_{\text {tuple }_{\text {body }}}\right\rangle \\
\text { tuple } & ::=\left\langle{\overline{\left(x_{\text {name }}: \text { cell }_{\text {value }}\right)}}_{\text {attributes }}, \text { form }_{\text {exists }}, \text { form }_{\text {at } \text { Cons }}\right\rangle \\
\text { cell } & ::=\text { atom } \mid \text { expr } \\
\text { domain } & ::=\mathrm{ID} \mid \mathrm{INT}
\end{array}
$$

Figure 5. Definition of rel as used in the translation. expr and form are defined in Figure 4.

some operator gives the following result:

$$
\begin{aligned}
& \left.T_{F} \text { [some Person where age }>=18\right] \rho \\
& =\bigvee\left(\begin{array}{ll|cc}
\mathrm{p} I d & \text { age } & \text { exists } & \text { attCons } \\
\hline \mathrm{p} 1 & 17 & b_{0} & \perp \\
\mathrm{p} 2 & i_{0} & b_{1} & i_{0} \geq 18
\end{array}\right) \\
& =\left(b_{0} \wedge \perp\right) \vee\left(b_{1} \wedge i_{0} \geq 18\right) \\
& =b_{1} \wedge i_{0} \geq 18
\end{aligned}
$$

The some formula is satisfied if at least one tuple in the relation exists. This is accomplished by translating it to a disjunction of the conjoined exists and attCons columns of the tuples in the relation (i.e. this is depicted by the big vee notation in the above translation).

As can be seen, the translation of this formula results in the SMT formula $b_{1} \wedge i_{0} \geq 18$. This means that an instance of this problem is satisfying iff it contains the second tuple (i.e. $b_{1}$ must be true) and the value of its age attribute is greater than or equal to 18 . The presence or absence of the first tuple does not change the validity of the resulting instance since it assigned age value of 17 never conforms to the formulated constraint. This means that a satisfying instance can either contain or not contain the first tuple as long as the second tuple is present.

\subsection{The Algorithm}

The translation of an AlleAlle specification starts with the translation of a problem using the function $T_{P}$ :

$$
\begin{aligned}
& T_{P}: \text { problem } \rightarrow \text { form } \\
& T_{P}\left[r_{1}\left(h_{1}\right) b_{1} \ldots r_{n}\left(h_{n}\right) b_{n} f_{1} \ldots f_{m}\right]=\bigwedge_{i=1}^{m} T_{F}\left[f_{i}\right] \rho \\
& \text { where } \rho=\bigcup_{j=1}^{n}\left(r_{j} \mapsto T_{R}\left[\left(h_{j}\right) b_{j}\right]\right)
\end{aligned}
$$

This function translates the constraints $f_{1} \ldots f_{m}$ of the problem to formulas (of type form, see Figure 4). The environment is populated using the $T_{R}$ function which converts relation declarations to the internal tabular representation. The $T_{P}$ function returns a conjunction of all the translated constraints.

The Relation data structure Central to the translation is the internal relation data structure, shown in Figure 5, which in this paper we visualize using the tabular notation introduced above. A relation rel consists of a header and 


$$
\begin{aligned}
& T_{R} \quad: \text { relHeader } \rightarrow \text { relBody } \rightarrow \text { rel } \\
& T_{R}[(h)=b] \quad=\operatorname{add}(\langle h, \varnothing\rangle, b, \lambda t .\langle\operatorname{convert}(t), \top, \top\rangle) \\
& T_{R}[(h)<=u b] \quad=\operatorname{add}(\langle h, \varnothing\rangle, u b, \lambda t .\langle\operatorname{convert}(t), x, T\rangle) \\
& T_{R}[(h)>=l b<=u b] \quad=\operatorname{add}(\langle h, \varnothing\rangle, u b, \lambda t \text {. }\langle\operatorname{convert}(t), \operatorname{exists}(t, l b), T\rangle) \\
& \text { add } \quad: \text { rel } \rightarrow \overline{\text { tupleDecl }} \rightarrow(\text { tupleDecl } \rightarrow \text { tuple }) \rightarrow \text { rel } \\
& \operatorname{add}[r, b, f] \quad=\text { if } b=\varnothing \text { then } r \text { else let } t \in b \text { in } \operatorname{add}(\operatorname{addDistinct}(r, f(t)), b \backslash t) \\
& \text { convert } \quad: \text { tupleDecl } \rightarrow \overline{x: \text { cell }} \\
& \text { convert }[t] \quad=\left\langle\left(\text { atr name }_{\text {nam }}:\left\{\begin{array}{l}
\text { id when } \text { atr }=i d \\
i \text { when } \text { atr }=\text { hole }(i \text { as fresh int var }) \quad \mid \text { atr } \in t)\rangle \\
\text { constant when } \text { atr }=\text { constant }
\end{array}\right.\right.\right. \\
& \text { exists } \quad: \text { tupleDecl } \rightarrow \text { bound } \rightarrow \text { form } \\
& \text { exists }[t, l b] \quad=\left\{\begin{array}{l}
\top \text { when } t \in l b \\
x \text { otherwise (with } x \text { as fresh bool var) }
\end{array}\right. \\
& \text { addDistinct } \quad: \text { rel } \rightarrow \text { tuple } \rightarrow \text { rel }
\end{aligned}
$$

Figure 6. Definition and construction of relations. The definition of the addDistinct function is included in Appendix A. relHeader, relBody, bound, tupleDecl, id and hole are declared in Figure 2. The other definitions are given in Figure 4 and Figure 5.

File $\mapsto$\begin{tabular}{lll|cc} 
old & depth & size & exists & attCons \\
\hline f0 & 2 & 100 & $\mathrm{~T}$ & $\mathrm{~T}$ \\
$\mathrm{f} 1$ & $i_{0}$ & $i_{1}$ & $b_{0}$ & $\mathrm{~T}$ \\
$\mathrm{f} 2$ & $i_{2}$ & $i_{3}$ & $b_{1}$ & $\mathrm{~T}$
\end{tabular}

Figure 7. The visual representation of File rel after its construction based on the relation declaration: File (oId:id, depth:int, size:int) $>=\{\langle f 0,2,100\rangle\}<=\{<$ $f 0,2,100>,<f 1, ?, ?>,<f 2, ?, ?>\}$.

a body. The header is defined as a mapping from attribute names to domains. The body is a set of tuples. Each tuple in the body contains the declared attributes and two additional columns, exists and attCons. When we refer to tuple we refer to the combination of the attributes and exists and attcons columns. In the translation rules we will use the subscripts $r_{\text {header }}$ and $r_{\text {body }}$ for the header and body of a relation $r$ and $t_{\text {attributes }}, t_{\text {exists }}$ and $t_{\text {attCons }}$ for the attributes, exists and attCons columns of a tuple $t$ to refer to the specific parts of a relation or tuple (see Figure 5).

\subsubsection{Constructing relations}

A rel can be constructed in three different ways depending on how it is declared. Figure 6 shows the definition of the construction function $T_{R}$. This function translates the relation definition (i.e. the header and lower and upper bounds) to a rel. Which construction function is used depends on how the bounds are declared and influences the value of the tuple's exists field. This value depends on whether the tuple declaration is part of the lower and upper bound or only of the upper bound. For instance, in the example of the small file system (Figure 1) the File relation is declared with both a lower and an upper bound (e.g. $>=\{<f \theta, 2,100\rangle\}<=\{<f \theta$ $, 2,100>,<f 1, ?, ?>,<f 2, ?, ?>\})$.

On construction of the rel the value $T$ is assigned to the exists field of the tuple $<f 0,2,100>$ since there cannot be a satisfying instance without this tuple present. It is a different case for the tuples $<f 1$, ? , ? $>$ and $<f 2$, ? ? ? $>$. Since they are only part of the relation's upper bound there may be satisfying instances where these tuples (or one of these tuples) are not present. To encode this, fresh boolean variables are assigned to the exists fields of both tuples. It is then up to the underlying SMT solver to find a satisfying assignment for these boolean variables. The full encoding of the File relation is shown in Figure 7 (with the exists column highlighted). The encoding is adapted from the relational model finder KoDKOD, with the difference that it is encoded in our relation data structure instead of a boolean matrix as used by KoDKoD [31].

Ensuring tuple distinctness The attCons column holds the constraints that were added on the tuple's scalar attributes. On construction this column will be populated with $T$ for most tuples. The only exception to this is when the added tuples in the relation are potentially non-distinct. Consider for instance the (valid) case that the File relation would have an upper bound of two possible tuples: $\langle f 1,1,10\rangle,<f 1$ , ?, ?>. Since they both share the same oid value (namely f1) these tuples could potentially overlap if the depth and size attributes of the second tuple would evaluate to 1 and 10 respectively.

Since the relational model dictates true set semantics for its relational bodies, the translation must enforce that all tuples in the relation are distinct, or collapsed into each other. To enforce this the translation algorithm adds a constraint to the attCons field of the second tuple that forces the value of either the depth or size attribute to be different, if the first tuple exists in the relation.

This distinctness rule is added on construction of the relations by applying the addDistinct function (included in Appendix A). This function checks whether tuples can potentially overlap and adds the necessary constraints to the attCons field. In the case of the example given in this section the constructed relation would be as follows:

File $\mapsto$\begin{tabular}{lll|cc} 
oid & depth & size & exists & attCons \\
\hline f1 & 1 & 10 & $b_{0}$ & T \\
& $i_{0}$ & $i_{1}$ & $b_{1}$ & $\neg b_{0} \vee \neg\left(i_{0}=1 \wedge i_{1}=10\right)$
\end{tabular}

\subsubsection{Translating AlleAlle constraints}

The entry for translating ALLEALle formulas to SMT formulas is the $T_{F}$ function, shown in Figure 8. To translate an AlleAlle expression the $T_{F}$ function calls the $T_{E}$ function which is defined in Figure 9. 


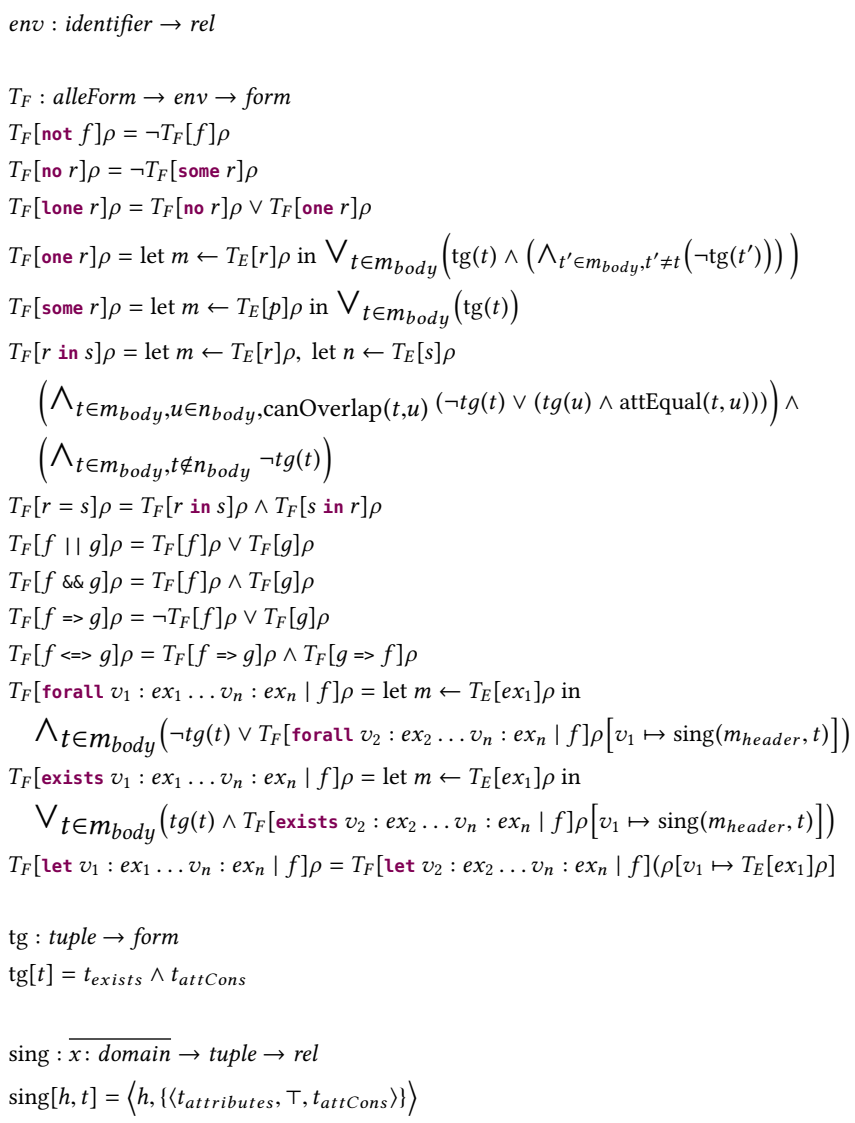

Figure 8. Translation rules for AlleAlle formulas. $r$ and $s$ are alleExpr, $f$ and $g$ are alleForm. Definitions of canOverlap and attEqual are included in Appendix A. $t g$ is short for together and sing is short for singleton meaning a relation with only one tuple.

The $T_{E}$ function translates the expression and returns a new rel. The $T_{F}$ function flattens the translated rels into SMT formulas. These in turn get conjoined by the $T_{P}$ function introduced earlier.

Tuple equality constraints When translating the subset formula and the union, intersection and difference expressions we again have to account for possible overlapping tuples described earlier. The difference being that in the case of translating the above rules we need to enforce equality instead of preventing it. In this case, the constraints that need to be added to the attCons field are constraints that force the value of the attributes to be the same; this is done using the helper functions canOverlap and attEquals, which are included in Appendix A.

As an example consider the following case. Suppose we have the following specification:

Shape (sid:id, size:int) $<=\{<s 1, ?>\}$

Square (sid:id, size:int) $<=\{<s 1, ?>\}$

Square in Shape

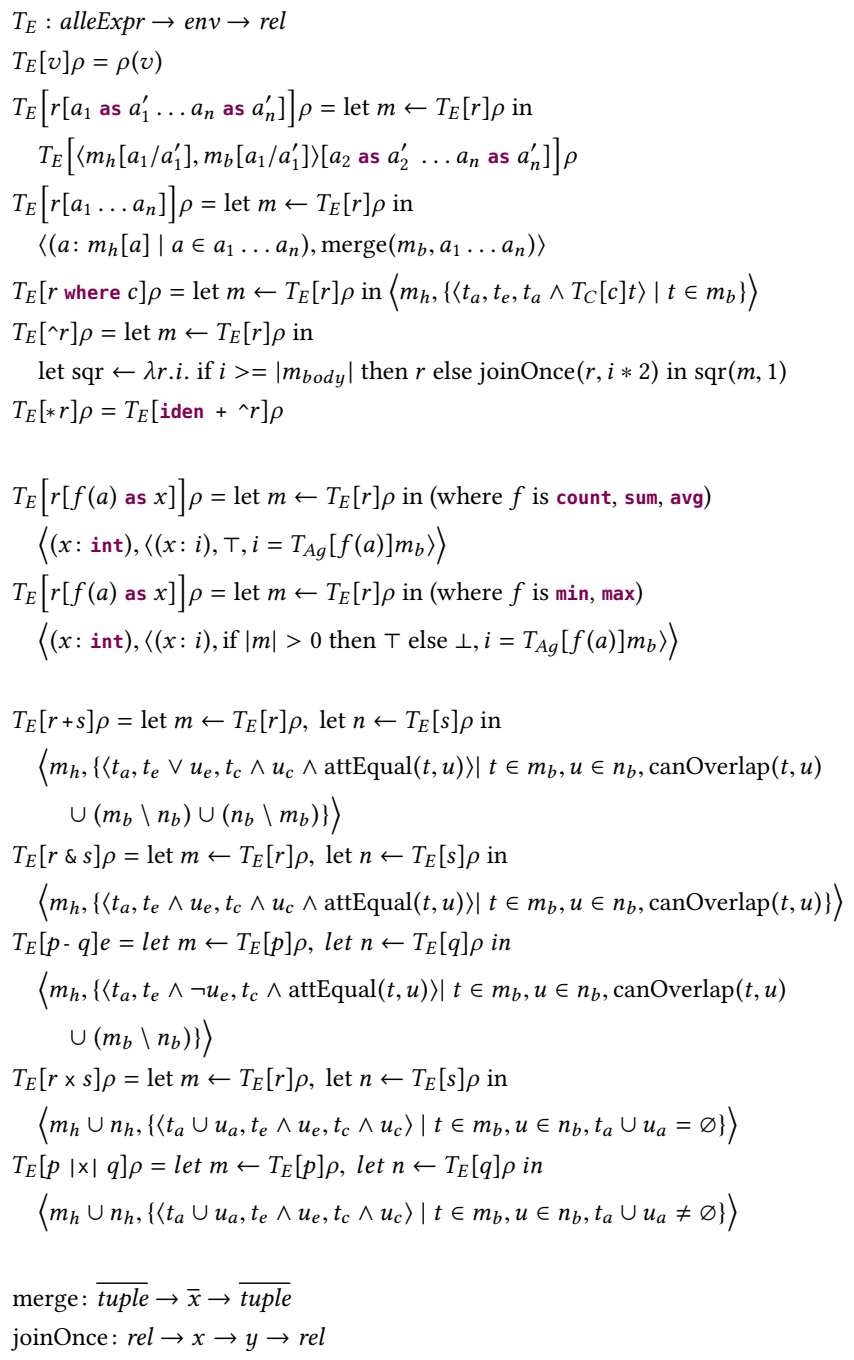

Figure 9. Translation rules for AlleAlle expressions. For abbreviation purposes the notation $r_{h}$ means $r_{h e a d e r}, r_{b}$ means $r_{\text {body }}, t_{a}$ means $t_{\text {attributes }}, t_{e}$ means $t_{\text {exists }}$ and $t_{c}$ means $t_{\text {attCons. }} r$ and $s$ are alleExpr, $c$ is a condition. Definitions of canOverlap and attEqual are included in Appendix A. The definition of merge and joinOnce is not given but sketched in the text. iden resolves to the binary identity relation on all values in the id domain.

We define two relations, Shape and Square. Both relations have an sid field of type id and a size field of type int. The sid fields contain the same id literal and both size attributes have been left open. Translating the constraint Square in shape would yield the following result:

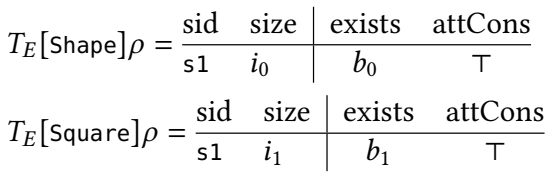

$T_{F}[$ Square in Shape $] \rho=\neg b_{1} \vee\left(b_{0} \wedge i_{0}=i_{1}\right)$ 


$$
\begin{array}{ll}
T_{C} & : \text { tuple } \rightarrow \text { form } \\
T_{C}[! c] t & =\neg T_{C}[c] t \\
T_{C}\left[c_{1} \& \& c_{2}\right] t & =T_{C}\left[c_{1}\right] t \wedge T_{C}\left[c_{2}\right] t \\
T_{C}\left[c_{1}|| c_{2}\right] t & =T_{C}\left[c_{1}\right] t \vee T_{C}\left[c_{2}\right] t \\
T_{C}\left[e_{1} \odot e_{2}\right] t & =T_{C e}\left[e_{1}\right] t \odot T_{C e}\left[e_{2}\right] t \\
& \\
T_{C e} & : \text { tuple } \rightarrow \operatorname{expr} \\
T_{C e}[x] t & =t_{\text {attributes }}[x] \\
T_{C e}[n] t & =n \\
T_{C e}[-e] t & =-T_{C e}[e] t \\
T_{C e}[|e|] t & =\text { let } i \leftarrow T_{C e}[e] t \text { in } i<0 ?-i: i \\
T_{C e}\left[e_{1} \oplus e_{2}\right] t & =T_{C e}\left[e_{1}\right] \oplus T_{C e}\left[e_{2}\right]
\end{array}
$$

Figure 10. Translation rules for the restriction conditions. $\odot$ depicts the different equality operators $(<,<=,=,=>,>), \oplus$ depicts the arithmetic operators $(+,-,,, /, \circ)$.

The outcome is that a Square can only be a Shape if either the square relation is empty (by enforcing that $b_{1}=\perp$ ) or the value of the size attributes of both relations is equal (by enforcing that $i_{0}=i_{1}$ ). Otherwise the tuple in Square would not overlap with the tuple in Shape and thus would not be in the subset relation.

Translation of the projection expression Projection can reduce the numbers of the tuples in the relation by truncating it. This again can potentially cause tuple overlap. Consider for instance the Person relation introduced in the translation example (Section 4.1). This relation has two attributes, pId and age and was defined as follows:

Person (pId:id, age:int) $<=\{<p 1,17>,<p 2, ?>\}$. resulting in the following internal representation:

Person $\mapsto$\begin{tabular}{cl|cc} 
pId & age & exists & attCons \\
\hline p1 & 17 & $b_{0}$ & $\mathrm{~T}$ \\
p2 & $i_{0}$ & $b_{1}$ & $\mathrm{~T}$
\end{tabular}

If we would project the age attribute both tuples could potentially collapse into each other. This would be the case if both tuples exist and the value of the age attribute of the second tuple would also be 17 . To prevent this the merge function adds extra constraints to the tuples reusing the addDistinct function (see Appendix A) enforcing that in all possible evaluations of its variables the result would be a relation with distinct tuples. We would end up with a relation with the following values and constraints:

$$
\text { Person } \mapsto \begin{array}{c|cc}
\text { age } & \text { exists } & \text { attCons } \\
\hline 17 & b_{0} & \top \\
i_{0} & b_{1} & \neg b_{0} \vee \neg\left(i_{0}=17\right)
\end{array}
$$

Translation of the aggregation expression Aggregation results in a new relation containing zero or one tuple with a single attribute. The value of the attcons field of this tuple

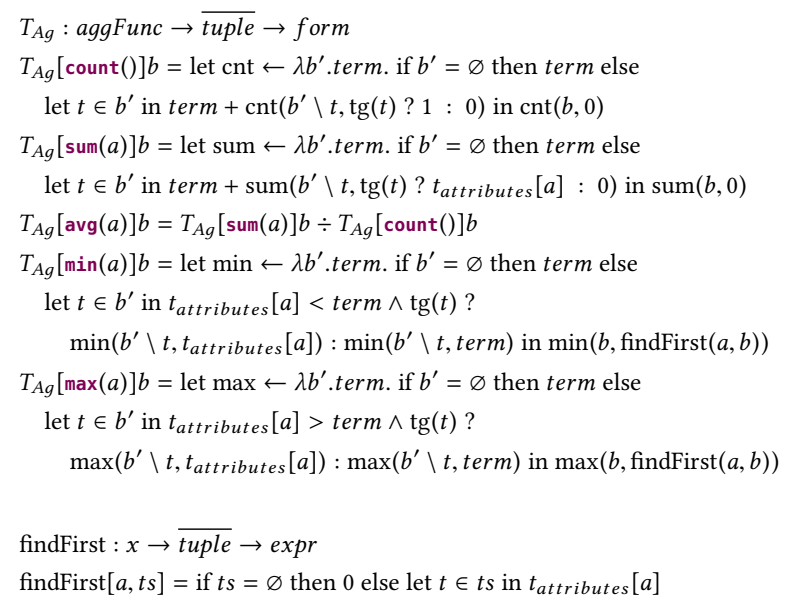

Figure 11. Translation rules for the aggregation functions

contains the unfolded aggregation expression. The translation of the different rules are shown in Figure 11.

In case of the application of the count, sum and avg aggregation the resulting relation will always contain a single tuple, even if the aggregated relation was empty. The intuition behind this is that even if the aggregated relation is empty its cardinality is zero and the sum of one of its attributes will also result in zero. The resulting relation after applying the min and max aggregation could be empty since calculating the max or min of an empty relation is undefined.

Translation of the transitive closure expression Transitive closure is only defined for binary relations containing two id attributes. In theory it is possible to define transitive closure for other data domains but the existence of possible holes would complicate the generation of the right equality constraints. Because of this reason we decided to postpone the calculation of transitive closure for other domain to possible future work.

In essence, calculating the transitive closure can be performed by recursively joining the relation with itself. To calculate the transitive closure we apply a variant of iterative squaring that works on our relation data structure. On every iteration the same translations are applied to the previously calculated relation. A single step of this translation is applied in the declared but not defined joinOnce function.

\subsection{Testing the Translation}

To gain more confidence in the correctness of the AlLEAlle translation we compared AlleAlle with KodKod [28] and the CHOco solver [23] on a number of different Constraint Satisfaction Problems (CSP) an Constraint Optimization Problems (COP). KоDKOD is also a relational model finder but is not able to solve optimization problems directly. The CHOco solver is able to solve optimization problems but its formalism and solving strategy is semantically further away from AlleAlle. 
Table 1. Testing AlleAlle against Kodkod and CHoco.

\begin{tabular}{|c|c|c|c|c|c|c|c|}
\hline Problem & $\begin{array}{l}\text { Problem } \\
\text { type }\end{array}$ & $\begin{array}{c}\text { Compare } \\
\text { with }\end{array}$ & $\begin{array}{c}\text { Sat. } \\
\text { ALLEALLE? }\end{array}$ & $\begin{array}{l}\text { Sat. } \\
\text { other? }\end{array}$ & $\begin{array}{c}\text { \#inst } \\
\text { AlleAlle }\end{array}$ & $\begin{array}{l}\text { \#inst } \\
\text { other }\end{array}$ & $\begin{array}{l}\text { Same opt. } \\
\text { solution? }\end{array}$ \\
\hline FileSystem & CSP & КоDKOD & Yes & Yes & 2184 & 2184 & - \\
\hline Handshake & CSP & KODKOD & Yes & Yes & 24 & 24 & - \\
\hline Pigeonhole & CSP & KоDKOD & No & No & - & - & - \\
\hline RingElection & CSP & КорKоD & Yes & Yes & 2 & 2 & - \\
\hline RiverCrossing & CSP & КоDKOD & Yes & Yes & 2 & 2 & - \\
\hline 8Queens & CSP & Сносо & Yes & Yes & 92 & 92 & - \\
\hline Sudoku & CSP & Сносо & Yes & Yes & 1 & 1 & - \\
\hline SendMoreMoney & CSP & Сносо & Yes & Yes & 1 & 1 & - \\
\hline Knapsack & $\mathrm{COP}$ & Сносо & Yes & Yes & - & - & Yes \\
\hline Mariokart & $\mathrm{COP}$ & Сносо & Yes & Yes & - & - & Yes \\
\hline
\end{tabular}

In the comparison of CSP problems we compare whether AlleAlle and Kodkod, or AlleAlle and Choco find the same answer, and whether they produce the same number of satisfying instances. When comparing COP problems we check whether both AlleAlle and CHoco find the same optimal solution. All problems are existing examples or benchmark problems from KoDKоD or $\mathrm{CHOCO}^{5}$. The results are shown in Table $1 .^{6}$

As can be seen in the results AlleAlle finds the same solutions as KоDKOD and CHOCo for all implemented problems. Please note that the reported found instances also contain all symmetric solutions. For instance the 8 queens problem has 92 solutions, but if symmetry is taken into account only 12 distinct solutions remain. KoDKOD can detect such symmetries by generating so called symmetry breaking predicates but for this benchmark we configured KoDKoD not to do this [28].

\section{Evaluation}

We evaluate ALlEAlle in terms of performance and expressiveness, by comparing the translation and solving times of AlleAlle with KoDKoD [28] on different problems, and by implementing a real-world use case, optimal dependency resolution [1], respectively. Finally, we qualitatively compare AlleAlle to similar systems for solving constraint problems.

\subsection{Translation and Solving Time Benchmark}

We compare AlleAlle's translation and solving time performance against the translation and solving time performance of KoDKоD by translating and solving six different problems. Table 2 characterizes the benchmark problems in terms of the kinds of constraints that are used.

For five of these problems we measure the performance for different configurations of the same problem to get insight in the effect of the size of a problem specification. Configuration parameters are the number of atoms or tuples that are allowed to populate the relations, and the allowed bit-width for the integer encoding used for KoDKoD.

\footnotetext{
${ }^{5}$ See https://github.com/chocoteam/samples/

${ }^{6}$ See https://github.com/joukestoel/allealle-benchmark/ for the encoding of the problems.
}

Table 2. Overview of the benchmarked problems.

\begin{tabular}{ll}
\hline Problem & Constraint types \\
\hline Alloy FileSystem & Relational \\
Halmos handshake & Relational, cardinality \\
Pigeonhole & Relational \\
River crossing puzzle & Relational \\
Square $y=x^{2}$ & Integer \\
Account state transition system & Relational, integer \\
\hline
\end{tabular}

The benchmarks are run on a early 2015 MacBook Pro with a 2,7 GHz quad core Intel i5 processor with $8 \mathrm{~GB}$ of DDR3 RAM. Java 8 (version 1.8.0_131 by Oracle) is used for all benchmarks. The translation and solving per configuration per problem was run 30 times with a warmup of 10 runs. All caches were flushed between each run. We report the median of the translation and solving times.

AlleAlle is implemented in RAscal [17]. RAscal is a functional programming language designed for the development and analysis of programming languages. It is an interpreted language that runs on the JVM. All AlleAlle benchmarks were run using version 0.12.0.201901101505 of RASCAL and version 4.8 .0 of $\mathrm{Z} 3$.

KoDKOD is implemented in Java and was built and run using the previously mentioned Java version. Some of the benchmarked problems contain cardinality and integer constraints. To avoid wrap-around semantics for integer constraints in KoDKoD we use KoDKoD* [20, 21], which is currently packaged with Alloy 4.2; the solver is configured with SAT4J (version 2.3.5.v20130525). As earlier, we configured KoDKOD to not generate symmetry breaking predicates.

Interpreting the results Table 3 contains the results of benchmarking AlleAlle against KoDKOD, comparing translation times and solving times. As can be seen in the results, AlleAlle is slower in translating purely relational problems (e.g., FileSystem, Pigeonhole and Rivercrossing). The reason for the slow-down is twofold. First, our current implementation of AlleAlle is a prototype, built as a proof-of-concept to demonstrate the correctness of the translation algorithm. Furthermore, AlleAlle is implemented in RASCAL, which is an interpreted language for language prototyping, whereas KoDKоD is implemented in Java. We are currently working on a Java implementation of AlleAlle which, we expect, will bring the translation performance up to par with KoDKOD since AllEAlle translation algorithms are of the same complexity as KoDKoD's translation.

AlleAlle is also slower in solving purely relational problems compared to KoDKoD. This can be explained from the fact that AlleAlle generates more clauses than KodKoD. For instance, for the FileSystem problem in the configuration with 30 atoms AlleAlle generates a total of 5359296 clauses while KoDKoD merely generates 24753 clauses. The reason for this difference is that KoDKOD implements a clause 
Table 3. Benchmark comparison between AlleAlle and KodKoD. Comparison shows six problems of which five are shown with different relation sizes, either in number of atoms or integer bit width used.

\begin{tabular}{|c|c|c|c|c|c|c|c|c|c|c|c|}
\hline \multirow[b]{2}{*}{ Problem } & \multirow[b]{2}{*}{ \#Atoms } & \multirow[b]{2}{*}{$\begin{array}{c}\text { Bit width } \\
\text { (KoDKOD only) }\end{array}$} & \multirow[b]{2}{*}{ Sat? } & \multicolumn{4}{|c|}{ AlleAlle } & \multicolumn{4}{|c|}{ КоDKоD } \\
\hline & & & & $\begin{array}{l}\text { Trans. time } \\
\quad \text { (in ms) }\end{array}$ & $\begin{array}{l}\text { Solve time } \\
\text { (in ms) }\end{array}$ & \#Vars & \#Clauses & $\begin{array}{l}\text { Trans. time } \\
\text { (in ms) }\end{array}$ & $\begin{array}{l}\text { Solve time } \\
\text { (in ms) }\end{array}$ & \#Vars & \#Clauses \\
\hline FileSystem ${ }^{r}$ & 15 & - & SAT & 725 & 20 & 130 & 21839 & 12 & 4 & 135 & 3235 \\
\hline FileSystem ${ }^{r}$ & 30 & - & SAT & 14567 & 110 & 460 & 5359296 & 29 & 8 & 470 & 24753 \\
\hline HandShake $^{r, c}$ & 10 & 4 & SAT & 480 & 10 & 184 & 1633 & 12 & 61 & 200 & 9292 \\
\hline HandShake $^{r, c}$ & 17 & 5 & UNSAT & 2235 & 69865 & 548 & 6295 & 23 & 136465 & 578 & 39214 \\
\hline Pigeonhole $^{r}$ & 9 & - & UNSAT & 51 & 10 & 20 & 302 & 2 & 1 & 20 & 137 \\
\hline Pigeonhole $^{r}$ & 17 & - & UNSAT & 77 & 1180 & 72 & 1424 & 1 & 45 & 72 & 565 \\
\hline Rivercrossing $^{r}$ & 12 & - & SAT & 327 & 10 & 74 & 1621 & 7 & 0 & 68 & 749 \\
\hline Square $^{i}$ & 2 & 4 & SAT & 5 & 10 & 2 & 6 & 2 & 4 & 36 & 3025 \\
\hline Square $^{i}$ & 2 & 10 & SAT & 5 & 10 & 2 & 6 & 198 & 11883 & 2052 & 371722 \\
\hline Account $^{r, i}$ & 12 & 5 & SAT & 72 & 10 & 33 & 289 & 21 & 79 & 351 & 19698 \\
\hline Account $^{r, i}$ & 12 & 9 & SAT & 71 & 10 & 33 & 289 & 460 & 34080 & 5151 & 480198 \\
\hline
\end{tabular}

Problem contains r) relational constraints, c) cardinality constraints, i) integer constraints

rewriting system that is much more aggressive than what is currently implemented in AlleAlle [28].

The results show, however, that AlleAlle's native handling of data for problems that contain both relational and integer constraints, pays off, both in translation times and solving times. As mentioned before, KoDKoD needs to specifically encode the possible integers up to the configured bitwidth. This is needed because it needs to encode integer constraints as part of the SAT formula so that the underlying SAT-solver can solve the problem. This results in more clauses in the generated SAT formula and thus higher translation and solving times. Since AlLEAlle does not require this explicit encoding but can use the solvers built-in reasoning power on different theories it does not suffer from the same performance penalty. Therefore its translation and solving times is consistent for the same problem even if larger integer values are required.

For problems which encode explicit cardinality constraints like the HandShake problem we also see better performance of AlLEAlle since relations with higher cardinality require a larger bit-width in KoDKOD. ${ }^{7}$

\subsection{Optimal Dependency Resolution}

To evaluate the expressiveness of AlLEAlLE we have implemented a solution to the optimal package resolution problem, which is common in package managers like NPM, APT, or MAVEN [1]. Figure 12 shows an example of a package resolution problem taken from Tucker et.al. [32]. The user asks to install package $a$; the package resolver needs to compute which packages to install or uninstall in such a way that all dependencies are satisfied and no conflicts are violated. This has been shown to be an NP-complete problem [11].

\footnotetext{
${ }^{7}$ Explicit meaning using KoDKoD's integer cast expression sum ( ) on relations and using integer arithmetic expressions to formulate cardinality constraints.
}

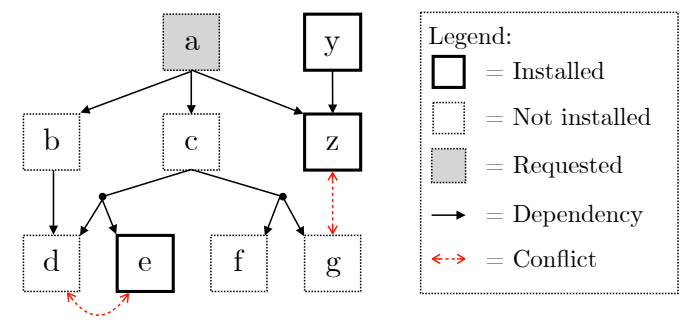

Figure 12. A package resolution problem (solution: install $\{a, b, c, d, f\}$ and uninstall $\{e\})$

We ran the experiment on the "paranoid" track of the MISC 2012 competition; a solver competition for package managers. ${ }^{8}$ This track required the contestants to find the minimal 'change' needed to the system to comply to the package update request. ${ }^{9}$ Because this problem requires to reason about the minimal change to the system it can not be directly encoded in ALLOY or KODKOD since these formalisms do not allow for the encoding of optimization problems.

All benchmarks were run on the same early 2015 MacBook Pro with a $2,7 \mathrm{GHz}$ quad core Intel i5 processor with $8 \mathrm{~GB}$ of DDR3 RAM. We compare our found results against the reported solutions and solving times from the MISC 2012 competition.

The package resolution problem can be compactly defined as a relational problem with data. The relevant relations are summarized in Table 4. The first 9 relations represent known facts about the package repository, what is installed on the user's system, and what the user requests to install, remove, or upgrade, respectively. These relations have exact bounds meaning that for every possible solution the tuples

\footnotetext{
${ }^{8}$ http://www.mancoosi.org/misc-2012/

${ }^{9}$ Minimal change meaning, minimal amount of packages that need to be removed and the minimal amount of packages needed to be installed or updated.
} 


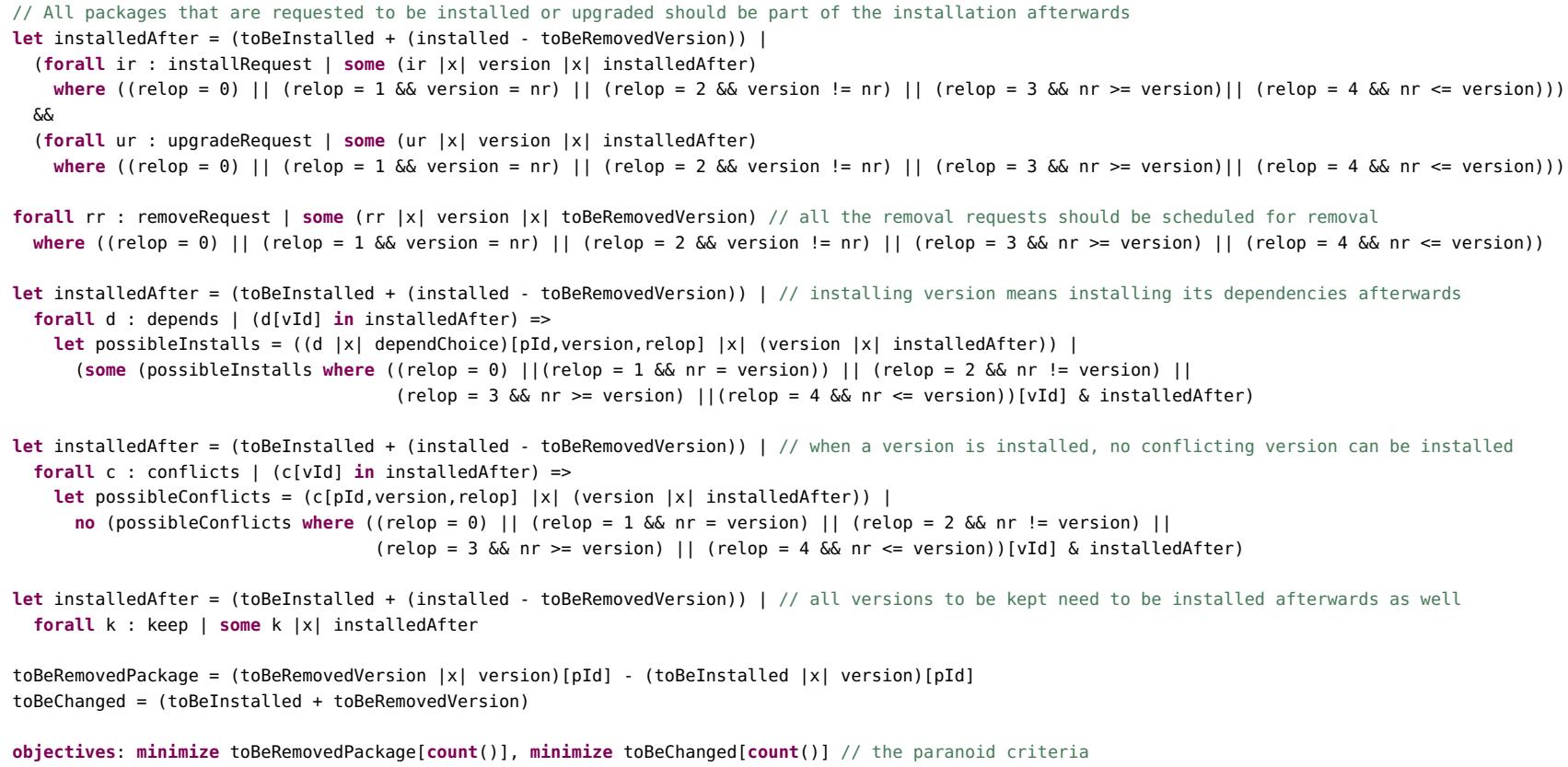

Listing 2. Optimal Package Resolution in AlleAlle

Table 4. Relations for the optimal package resolution problem

\begin{tabular}{lll}
\hline Relation & Signature & Bound \\
\hline installRequest & pId:id, relop:int, version:int & Exact \\
removeRequest & pId:id, relop:int, version:int & Exact \\
upgradeRequest & pId:id, relop:int, version:int & Exact \\
version & vId:id, pId:id, nr: int & Exact \\
installed & vId:id & Exact \\
keep & kId:id, vId:id & Exact \\
depends & vId:id, dcId:id & Exact \\
dependChoice & dcId:id, pId:id, version:int, relop:int & Exact \\
conflicts & vId:id, pId:id, version:int, relop:int & Exact \\
\hline toBeInstalled & vId:id & Upper \\
toBeRemovedVersion & vId:id & Upper \\
toBeChanged & vId:id & Upper \\
toBeRemovedPackage & pId:id & Upper \\
\hline
\end{tabular}

of these relations are exactly those that are defined in the specification. The other 4 relations have an upper bound and represent the solution space for the solver to satisfy the user's request, given dependency and conflict constraints between package versions. The rest of the specification is shown in Listing 2, consisting of a mere 30 source lines of AlleAlle code.

In total we translated 57 of the competition problems, and measured the time spent in translating AlleAlle and running $\mathrm{Z} 3$ to solve the constraints. All found solutions by AlleAlle were correct and optimal, showing that the constructed specification is a correct implementation of the optimal dependency resolution problem according to the paranoid criteria. The full results can be found in Table 5, in Appendix B.

The results show that $\mathrm{Z} 3$ can efficiently solve the formula produced by AlLEALLE, in the same order of magnitude as the winning solving times from the 2012 MISC competition. On the other hand, the time spent by AlleAlle translating the specification to SMT formula is high, ranging from 47 seconds to 62 minutes. Based on the specific problems exhibiting this behavior, we hypothesize that translation time correlates with the number of dependencies between the packages. The more dependencies between packages, the longer it takes AlLEAlle to translate the problem to SMT formulas.

\subsection{Comparing AlleAlle to Similar Systems}

AlleAlle is a constraint solving system and language, comparable to Kodkod, Alloy, and SMT solvers. AlleAlle is an intermediate language: it is higher-level than the first-order logic formulas of SMT solvers like Z3 [22] or CVC4 [5] which are more general purpose logic solvers, but lower-level than, e.g., Alloy, which is a end-user, modeling language.

KоDKOD is the back-end framework of Alloy, which is at the same level of abstraction as AlleAlle. Both AlleAlle and KoDKoD support relational constraints, yet AlleAlle employs Codd's relational algebra making it possible to constraint data attributes directly (using the where operator), whereas KoDKoD is based on Tarski's relation logic allowing constraints only to be expressed on the level of relations.

Although in terms of abstraction level, AlleAlle is comparable to KoDKOD, the latter only exists as a Java library and does not feature a concrete syntax. AlleAlle's syntax allows us to experiment with specifications in a more flexible way, and thus may function as code generation target for higher-level languages, - essentially fulfilling the same role that KoDKoD fulfills for Alloy. 
AlleAlle leverages built-in theories of underlying SMT solvers, including support for optimization criteria available in solvers like Z3 [7]. KoDKoD (and hence AlLOY) require bitencoding of integers because of their underlying SAT solvers, which is an impediment to performance for constraint problems that require such constraints. Optimization criteria are not available in either Alloy or KoDKOD. For instance, the optimal dependency resolution problem (Section 5.2) cannot be expressed in either Alloy or KodKoD.

AlleAlle thus occupies a sweet spot in terms of both expressiveness (Codd's algebra) and solving performance (because of native SMT theories) between high-level languages like Alloy, and low-level relational solvers like KoDKOD.

\section{Related Work}

SEM- and MACE-style model finders There are several finite model finding tools for first order logic (FOL). They can roughly be divided into two different groups: tools that implement specialized search strategies to find satisfying models (also known as SEM-style model finders) [27, 34] and tools that translate FOL formulas to SAT or SMT formulas and use an off-the-shelf solver to find satisfying instances (also known as MACE-style model finder) [9, 18, 25, 31]. AlLEALLe falls in the MACE-style category.

Although all of these model finders accept FOL formulas as input not many of them accept relational logic. SEM [34] and FINDER [27] for instance accept a many-sorted logic of uninterpreted functions but no quantifiers. MACE2 [18], PARADOX [9], Fortress [33] and Razor [25] do allow for quantifiers but do not offer direct support for relational expressions.

Razor opts for a slightly different angle where it focuses on model exploration by searching for a minimal model first and allowing for model exploration using a predefined preorder relation. To achieve this Razor also exploits the SMT solver Z3 [25].

Fortress [33] also exploits an SMT solver by mapping FOL formulas to the logic of equality with uninterpreted functions (EUF). To make the model finite Vakilie et al. introduce socalled range functions to force restriction on the number of elements assigned to the different sorts [33].

KoDKoD [31], the model finding engine used by Alloy [15, 30], accepts relational logic and transitive closure but offers no support for optimization criteria or first-class reasoning over data. As shown in the evaluation (see Section 5) KoDKOD and Alloy do support integers but require the user to specify a fixed bit-width. KoDKOD uses an explicit integer encoding which results in the introduction of more variables and ultimately into more CNF clauses in the generated SAT formula.

The other main difference with KoDKOD is the interpretation of relational logic: KODKOD uses Tarski's definition of relational logic, AllEAlle uses Codd's relational algebra.
The switch from Tarski to Codd prevents the need for an explicit encoding of data variables (i.e. integers) in the problem domain. E.g. in AlleAlle it is not needed to explicitly define the set of integers to be used in the relational expressions, it can directly encode constraints on the data attributes (using the where operator).

Relational reasoning with SMT El Ghazi et al. translate AlLoy specifications to unbounded SMT constraints using an axiomatization of ALLOY's relational logic in SMT, a so called shallow embedding of a relational theory [13]. Although this allows for proving some ALLOY specifications it also struggles with many specifications, as is shown in later work [19].

Meng et al. define a relational calculus based on the theory of finite sets with cardinality constraints [4, 19]. This calculus is created explicitly to be implemented as a new theory in SMT solvers, a so called deep embedding, and contains many of the relational expressions that are also part of KoDKoD (like join, transpose, product and transitive closure). The calculus does not require that bounds are set on the relations but uses earlier work by the authors on finite model finding [24]. The authors implement the calculus as a new theory in the SMT solver CVC4 [5] and evaluate its performance on existing benchmarks. Contrary to AlleAlle, the new theory is able to prove when a relational problem is unsatisfiable. Like Alloy and KodKod, AlleAlle requires bounds on the relations with the result that any reported unsat only means that it is unsatisfiable with respect to the given bounds. The work by Meng et al. is based on the same calculus as KoDKoD (Tarski's relational logic) meaning that reasoning on values of other theories (like integers) is limited in the same way as it is limited in KoDKoD. Next to that, this work is implemented in CVC4 which currently does not support optimization objectives.

\section{Conclusion}

Relational model finding is a powerful technique that can be applied to a wide range of problems. In this paper we have introduced AlleAlle, a new language for describing such problems with first-class support for data attributes. AllEAlle specifications combine first-order logic with Codd's relational algebra, transitive closure, and optimization objectives. We have presented the formal semantics of AlLEAllE and a detailed exposition of a novel algorithm to translate AlleAlle specifications to SMT constraints, to be solved by standard SMT solvers such as Z3.

Initial evaluation of our prototype implementation has shown that the performance leaves room for improvement. Both the translation speed and the generated formula efficiency can be improved. Theoretically it should be possible to improve the translation and solving time of pure relational problems to the level of KoDKOD's performance since the translation algorithm of both model finders is comparable in terms of complexity. Early performance experiments 
with a pure Java version of ALLEALLE indeed hint into this direction.

AlleAlle supports compact encodings of relational problems with data. One example is optimal dependency resolution, as used in package managers. We have used this problem to assess expressiveness of AlleAlle. Although currently the translation times are high for these problems, the resulting SMT formulas can be solved efficiently by offthe-shelf solvers. The specification itself is concise, taking only half a page, which shows the expressiveness of combining relational logic, data, and optimization criteria.

There are multiple directions for future work. The current prototype of ALLEALLE only supports integer domains; we will extend AlleAlle with support for reals, strings, and bit vectors since most SMT solves have built-in theories for these types. Another direction for further work is to include symmetry breaking predicates [9] to the generated SMT formulas, since this is well-known to have a positive effect on solver performance. Torlak et al. introduce a method to create these symmetry breaking predicates for relational logic [31], but it is an open question how this should be done in the presence of AlleAlle's data attributes. A final direction for further research is to investigate how unsatisfiability core extraction [29] can be used to explain reasons for unsat results. A particular challenge is how to map such explanations back to the level of AlLEAlle.

\section{A Algorithms}

addDistinct The function ADDDistinct adds a tuple to a relation, ensuring that it will be distinct from other tuples in the relation by adding constraints to the attCons column:

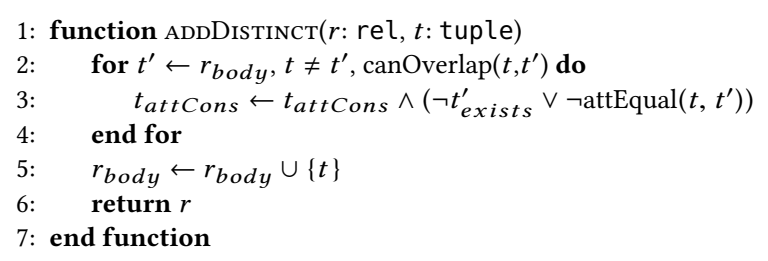

canOverlap The function CANOvERLAP returns $T$ when two tuples are indistinguishable with respect to their attribute values:

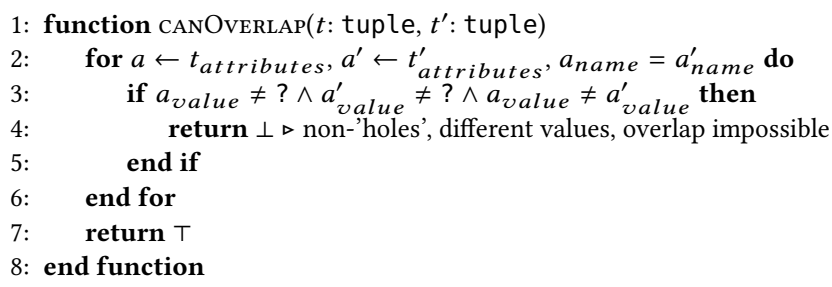

attEqual The function ATTEQUAL constructs a constraint to ensure that two tuples will be equal.

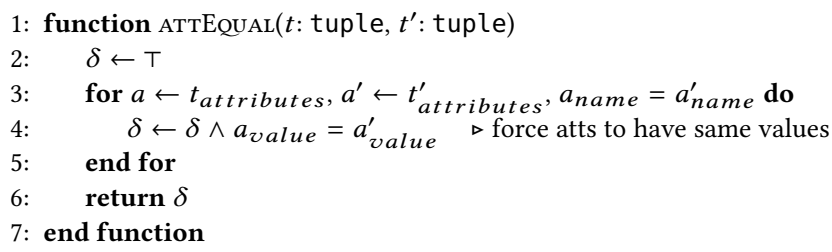

\section{B Optimal Package Dependency Resolution}

Table 5. "MISC paranoid" AlLeAlle results. Problem names refer to the problems as they were named in the original competition. See http://www.mancoosi.org/misc-2012/results/ paranoid/ for an overview.

\begin{tabular}{|c|c|c|c|c|c|c|c|c|}
\hline Problem name & 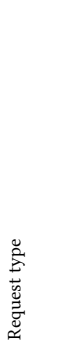 & 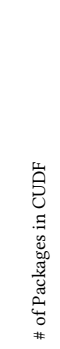 & 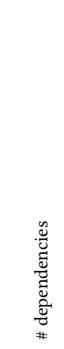 & 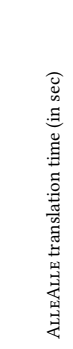 & 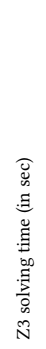 & 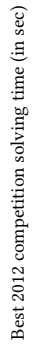 & $\begin{array}{l}\text { 苞 } \\
\text { : } \\
0\end{array}$ & 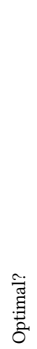 \\
\hline $163 \mathrm{e} 46 \mathrm{~d} 37 \mathrm{a}$ & upgrade & 203 & 2721 & 4.93 & 2.88 & 1.30 & yes & \\
\hline $\mathrm{d} 67-56 \mathrm{~d} 0-11 \mathrm{C}-2$ & 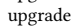 & 333 & 14170 & 343.96 & 2.29 & 0.63 & yes & \\
\hline e2f6303a-4fe9-11e0-aa4f-00163e & instal & 41913 & 29726 & 1077.47 & 1.72 & 2.57 & yes & \\
\hline 29180036-5408-11df-9f57-00163e7a6f5e & upgrade & 28753 & 17490 & 403.31 & 1.22 & 0.99 & yes & es \\
\hline $7 \mathrm{bf5} 0 \mathrm{~d} 1 \mathrm{c}-9 \mathrm{~b} 1 \mathrm{~b}-$ & Nomade & 47210 & 17934 & 459.80 & 3.35 & 1.32 & yes & \\
\hline 8b0e7c16-bab4-11e0-a 88 & install & 59100 & 57811 & 2879.15 & 4.20 & 2.60 & yes & yes \\
\hline 5698a62c-c731-11df-9bb9- & install & 54474 & 62705 & 3463.34 & 4.71 & 2.80 & yes & yes \\
\hline 6b0d1da0-c730-1 & install & 54474 & 62792 & 3609.27 & 5.86 & 1.62 & yes & \\
\hline 19890cfe-db9f-11df-9e6c-0016 & upgrade & 33615 & 23979 & 743.49 & 3.24 & 1.15 & yes & yes \\
\hline 978532fa-c730-1 & install & 54474 & 62792 & 25.55 & 4.50 & 2.80 & yes & \\
\hline $\mathrm{dd} 08 \mathrm{c}$ & install & 49561 & 48746 & 30.60 & 3.20 & 1.78 & yes & \\
\hline $9 \mathrm{a} 24-0016$ & install & 49561 & 48746 & 2327.53 & 3.68 & 1.44 & yes & es \\
\hline dba3a & un & 6310 & 17891 & 189.83 & 1.18 & 0.79 & yes & \\
\hline f4ebf $9 \mathrm{e} 0-3$ & grade & 213 & 18015 & 464.63 & 1.21 & 1.80 & yes & yes \\
\hline 4ede & & 849 & 40000 & 1630.78 & 2.45 & 1.47 & yes & yes \\
\hline & & 134 & 15370 & 336.95 & 0.73 & 51 & yes & yes \\
\hline ab9005be-bacc-11e0-b & inst & 100 & 57811 & 2914.14 & 4.15 & 47 & yes & es \\
\hline ff4a1 & ins & 540 & 53307 & 2519.30 & 2.40 & 2.26 & yes & es \\
\hline fa3d & & 3615 & 23979 & 726.63 & 3.14 & 0.89 & yes & yes \\
\hline 80e3fda2-9501-11e0-8001-00163e1e087d & install & 1134 & 15370 & 342.71 & 0.74 & 57 & es & yes \\
\hline d023 & & & 891 & 445.69 & 2.57 & 1.10 & yes & \\
\hline $103 \mathrm{c}$ & & 8753 & 4990 & 40.41 & 1.21 & 0.76 & yes & yes \\
\hline 4a69cf16-c731-11df-9182-00163e3d3b7c & at & 4474 & 62705 & 3761.11 & 4.74 & 1.62 & 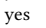 & es \\
\hline$c-00163 e$ & ins & 49561 & 48746 & 2272.93 & 3.28 & 1.65 & yes & \\
\hline & & 39025 & 21410 & 575.76 & 1.33 & 1.50 & yes & es \\
\hline cff22854-9512-11e0-8001-00163e1e087d & ll & 51134 & 15370 & 337.73 & 0.67 & 1.20 & yes & es \\
\hline 8680 & ast & 360 & 51425 & 2427.60 & 3.92 & 1.92 & yes & \\
\hline & & 10 & 891 & 429.89 & 1.25 & 1.26 & yes & es \\
\hline & & 34 & 370 & 349.90 & 0.79 & 1.54 & yes & es \\
\hline & or & 33615 & 3979 & 696.62 & 2.81 & 93 & yes & \\
\hline $\mathrm{ca}$ & e & 615 & 23979 & 860.79 & 2.81 & 0.82 & yes & s \\
\hline & & 13 & 015 & 466.59 & 1.26 & 1.14 & yes & es \\
\hline $\mathrm{d} 0 \mathrm{cc}$ & sta & 474 & 2705 & 3392.81 & 4.50 & 90 & yes & \\
\hline bccf & rrad & 615 & 979 & 82.21 & 2.81 & 96 & yes & es \\
\hline & & & 62817 & 3393.98 & 4.14 & 4 & yes & es \\
\hline & & 310 & 891 & 448.12 & 1.20 & 94 & yes & \\
\hline e69a & & 26 & 63860 & 82.47 & 4.58 & 1.68 & yes & es \\
\hline & & & 40000 & 59.44 & 2.21 & 1.26 & es & \\
\hline & & 94 & 9996 & 948.50 & 1.32 & 1.66 & es & \\
\hline a75 & & 134 & 15370 & 322.43 & 0.64 & 1.12 & yes & es \\
\hline & & & 4085 & 47.15 & 3 & & & \\
\hline & & 61 & 48746 & 09.19 & 20 & 50 & es & \\
\hline & & & & 45 & 3.40 & 0.95 & yes & es \\
\hline & 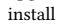 & 104 & 20996 & .50 & 1.39 & 1.72 & ves & \\
\hline eeee & grade & 28753 & 17490 & 410.22 & 1.22 & 0.63 & es & \\
\hline & & 47210 & 17934 & 420.83 & 1.74 & 1.32 & yes & es \\
\hline & & & & 2685.60 & 2.88 & 14 & yes & \\
\hline ' & graue & 47203 & 17933 & 422.87 & 70 & 1.22 & yes & \\
\hline & & 56865 & 55542 & 2798.53 & 0.00 & 00 & yes & es \\
\hline & & & & 951.58 & 16 & 58 & & \\
\hline $3 e 4 f$ & & & 4085 & 60.48 & 0.68 & 1.27 & s & \\
\hline & & 04 & 29996 & 7.15 & 1.31 & 1.66 & es & \\
\hline & & & 542 & 2785.50 & 0 & 00 & es & \\
\hline 4f0-4fe9-11e0-acd7-00163e1e087d & in & 41913 & 29720 & 897.63 & 55 & 1.79 & yes & s \\
\hline & & & 11545 & 5.60 & 0.33 & 1.11 & yes & \\
\hline 0207e19a-9b1c-11df-af69-00163e46d37a & grade & 47210 & 22707 & 571.12 & 2.67 & 1.30 & yes & \\
\hline
\end{tabular}

\section{Acknowledgments}

We would like to thank our sheperd and reviewers for their constructive feedback which helped to improve this paper. 


\section{References}

[1] P. Abate, R. Treinen, R. Di Cosmo, and S. Zacchiroli. MPM : a modular package manager. In CBSE, pages 179--188. ACM, 2011.

[2] A.V. Aho and J.D. Ullman. Universality of data retrieval languages. In POPL, pages 110-119. ACM, 1979.

[3] B. Bajić-Bizumić, C. Petitpierre, H.C. Huynh, and A. Wegmann. A model-driven environment for service design, simulation and prototyping. In ESS, pages 200-214. Springer, 2013.

[4] K. Bansal, A. Reynolds, C. Barrett, and C. Tinelli. A new decision procedure for finite sets and cardinality constraints in smt. In $C A V$, pages 82-98. Springer, 2016.

[5] C. Barrett, C.L. Conway, M. Deters, L. Hadarean, Jovanović. D., T. King, A. Reynolds, and C. Tinelli. CVC4. In Ganesh Gopalakrishnan and Shaz Qadeer, editors, CAV, volume 6806 of Lecture Notes in Computer Science, pages 171-177. Springer, July 2011.

[6] C. Barrett, A. Stump, C. Tinelli, et al. The smt-lib standard: Version 2.0. In SMT, volume 13, page 14, 2010.

[7] N. Bjørner, A. Phan, and L. Fleckenstein. $v Z$ - An Optimizing SMT Solver. In TACAS, volume 15, pages 194-199, 2015.

[8] J. Brunel, D. Chemouil, L. Rioux, M. Bakkali, and F. Vallée. A viewpointbased approach for formal safety \& security assessment of system architectures. In MoDeVVa, volume 1235, pages 39-48, 2014.

[9] K. Claessen and N. Sörensson. New techniques that improve macestyle finite model finding. In CADE-19 Workshop: Model ComputationPrinciples, Algorithms, Applications, pages 11-27. Citeseer, 2003.

[10] E.F. Codd. A relational model of data for large shared data banks. Communications of the ACM, 13(6):377-387, 1970.

[11] R. Di Cosmo. EDOS deliverable WP2-D2 . 1 : Report on Formal Management of Software Dependencies. Technical report, 2005. URL: https://hal.inria.fr/hal-00697463/document.

[12] C.J. Date. An Introduction to Database Systems. Reading, MA, AddisonWesley, 6th edition, 1994.

[13] A.A. El Ghazi and M. Taghdiri. Relational reasoning via smt solving. In FM, pages 133-148. Springer, 2011.

[14] D. Grunwald, C. Gladisch, T. Liu, M. Taghdiri, and S. Tyszberowicz. Generating jml specifications from alloy expressions. In $H V C$, pages 99-115. Springer, 2014.

[15] D. Jackson. Software Abstractions - Logic, Language, and Analysis. MIT press, revised edition, 2012.

[16] S.A. Khalek, G. Yang, L. Zhang, D. Marinov, and S. Khurshid. Testera: A tool for testing java programs using Alloy specifications. In ASE, pages 608-611. IEEE Computer Society, 2011.
[17] P. Klint, T. van der Storm, and J. Vinju. RASCAL: A Domain Specific Language for Source Code Analysis and Manipulation. In SCAM, pages 168-177. IEEE, 2009.

[18] W. McCune. A davis-putnam program and its application to finite first-order model search: Quasigroup existence problems. Technical report, Technical report, Argonne National Laboratory, 1994.

[19] B. Meng, A. Reynolds, C. Tinelli, and Clark Barrett. Relational constraint solving in smt. In $C A V$, pages 148-165. Springer, 2017.

[20] A. Milicevic and D. Jackson. Preventing arithmetic overflows in Alloy. Science of Computer Programming, 94:203-216, 2014.

[21] A. Milicevic, J.P. Near, E. Kang, and D. Jackson. Alloy*: a generalpurpose higher-order relational constraint solver. In ICSE, pages 609619. IEEE Press, 2015.

[22] L. De Moura and N. Bjorner. Z3: An efficient SMT solver. TACAS, pages 337-340, 2008.

[23] C. Prud'homme, J.G. Fages, and X. Lorca. Choco Documentation, 2017. URL: http://www.choco-solver.org.

[24] A. Reynolds, C. Tinelli, A. Goel, and S. Krstić. Finite model finding in smt. In $C A V$, pages 640-655. Springer, 2013.

[25] S. Saghafi. A Framework for Exploring Finite Models. PhD thesis, Worcester Polytechnic Institute, 2015.

[26] R. Sebastiani and P. Trentin. Optimathsat: a tool for optimization modulo theories. In $C A V$, pages 447-454. Springer, 2015.

[27] J. Slaney. Finder: Finite domain enumerator system description. In $C A V$, pages 798-801. Springer, 1994.

[28] E. Torlak. A Constraint Solver for Software Engineering : Finding Models and Cores of Large Relational Specifications. $\mathrm{PhD}$ thesis, Massachusetts Institute of Technology, 2009.

[29] E. Torlak, F. Chang, and D. Jackson. Finding minimal unsatisfiable cores of declarative specifications. In $F M$, pages 326-341. Springer, 2008.

[30] E. Torlak and G. Dennis. Kodkod for Alloy users. In Alloy Workshop, 2006.

[31] E. Torlak and D. Jackson. Kodkod: A relational model finder. In TACAS, pages 632-647. Springer, 2007.

[32] C. Tucker, D. Shuffelton, R. Jhala, and S. Lerner. Opium: Optimal package install/uninstall manager. In ICSE, pages 178-188. IEEE Computer Society, 2007.

[33] A. Vakili and N.A. Day. Finite model finding using the logic of equality with uninterpreted functions. In FM, pages 677-693. Springer, 2016.

[34] J. Zhang and H. Zhang. Sem: a system for enumerating models. In IFCAI, volume 95, pages 298-303, 1995. 